\title{
Fermion spectrum and $g-2$ anomalies in a low scale 3-3-1 model
}

\author{
A. E. Cárcamo Hernández ${ }^{1, \mathrm{a}}$, Yocelyne Hidalgo Velásquez ${ }^{1, \mathrm{~b}}$, Sergey Kovalenko ${ }^{2, \mathrm{c}}$, H. N. Long ${ }^{4,3, \mathrm{~d}}$, Nicolás A. \\ Pérez-Julve $^{1, \mathrm{e}}$, V. V. Vien ${ }^{5,6, f}$ \\ ${ }^{1}$ Universidad Técnica Federico Santa María and Centro Científico-Tecnológico de Valparaíso, Casilla 110-V, Valparaiso, Chile \\ ${ }^{2}$ Departamento de Ciencias Físicas, Universidad Andres Bello, Sazié 2212, Piso 7, Santiago, Chile \\ 3 Theoretical Particle Physics and Cosmology Research Group, Advanced Institute of Materials Science, Ton Duc Thang University, \\ Ho Chi Minh City, Vietnam \\ ${ }^{4}$ Faculty of Applied Sciences, Ton Duc Thang University, Ho Chi Minh City, Vietnam \\ ${ }^{5}$ Institute of Research and Development, Duy Tan University, 182 Nguyen Van Linh, Da Nang, Vietnam \\ ${ }^{6}$ Department of Physics, Tay Nguyen University, 567 Le Duan, Buon Ma Thuot, DakLak, Vietnam
}

Received: 25 February 2020 / Accepted: 13 February 2021 / Published online: 26 February 2021

(C) The Author(s) 2021

\begin{abstract}
We propose a renormalizable theory based on the $S U(3)_{C} \times S U(3)_{L} \times U(1)_{X}$ gauge symmetry, supplemented by the spontaneously broken $U(1)_{L_{g}}$ global lepton number symmetry and the $S_{3} \times Z_{2}$ discrete group, which successfully describes the observed SM fermion mass and mixing hierarchy. In our model the top and exotic quarks get tree level masses, whereas the bottom, charm and strange quarks as well as the tau and muon leptons obtain their masses from a tree level Universal seesaw mechanism thanks to their mixing with charged exotic vector like fermions. The masses for the first generation SM charged fermions are generated from a radiative seesaw mechanism at one loop level. The light active neutrino masses are produced from a loop level radiative seesaw mechanism. Our model successfully accommodates the experimental values for electron and muon anomalous magnetic dipole moments.
\end{abstract}

\section{Introduction}

Despite of the excellent agreement of the Standard Model (SM) predictions with the experimental data, there are several problems that do not find explanation within its framework. Among them are the observed pattern of SM fermion masses and mixing angles, the tiny values of the light active neutrino masses, the number of SM fermion families, the

\footnotetext{
a e-mail: antonio.carcamo@usm.cl

b e-mail: yocehidalgov@gmail.com

c e-mail: sergey.kovalenko@usm.cl

d e-mail: hoangngoclong@tdtu.edu.vn (corresponding author)

e e-mail: nicolasperezjulve@gmail.com

fe-mail: wvienk16@gmail.com
}

electric charge quantization and the anomalous magnetic moments of the muon and electron. Addressing these issues requires to consider extensions of the SM with enlarged particle content and symmetries. In particular, theories based on the $S U(3)_{C} \times S U(3)_{L} \times U(1)_{X}$ gauge symmetry (3-3-1 models) [1-52], have received a lot of attention since they answer some of the open questions of the SM, such as, for example, the number of SM fermion families and the electric charge quantization. Adding discrete symmetries and extending the scalar and fermionic content of such 3-3-1 models allows addressing the observed SM fermion mass and mixing hierarchy. Furthermore, if one considers 3-3-1 models where the fermions do not have exotic electric charges, the third component of the $S U(3)_{L}$ leptonic triplet will be electrically neutral. This allows the implementation of a low scale linear or inverse seesaw mechanism producing the tiny light active neutrino masses and sterile neutrinos with masses at the $S U(3)_{L} \times U(1)_{X}$ symmetry breaking scale, thus making the model testable at colliders.

Imposing discrete symmetries allows one to forbid tree level masses arising from the Standard Yukawa interactions for the SM fermions lighter than the top quark. To generate such masses, one has to consider heavy vector-like fermions, mixing with the SM fermions lighter than the top quark, as well as gauge singlet scalar fields. Their inclusion in the particle spectrum of the model is crucial for the implementation of the Universal and radiative seesaw mechanisms needed to generate the masses for the SM fermions lighter than the top quark, thus explaining the SM charged fermion mass hierarchy. In addition, the heavy vector like leptons can provide an explanation for the anomalous electron and muon magnetic moments, which is not given within the context of the SM. A study of such $g-2$ anomalies in terms of New Physics 
and a possible UV complete explanation via vector-like leptons is performed in [53]. Also in Ref. [54], it was shown that the $g-2$ anomalies can be explained using a minimal supersymmetric SM assuming a minimal flavor violation in the lepton sector. Theories involving extended scalar sector [53-70] as well as vector like leptons [71], heavy $Z^{\prime}$ gauge bosons [51,72], and conformal extended technicolour [73] have been proposed to explain the $g-2$ anomalies. In this work we will consider a renormalizable theory based on the $S U(3)_{C} \times S U(3)_{L} \times U(1)_{X}$ gauge symmetry, supplemented by the spontaneously broken $U(1)_{L_{g}}$ global lepton number symmetry and the $S_{3} \times Z_{2}$ discrete group. We choose $S_{3}$ symmetry since it is the smallest non-Abelian discrete symmetry group having three irreducible representations (irreps), explicitly, two singlets and one doublet irreps. This symmetry has been shown to be useful in several extensions of the $\mathrm{SM}$, for obtaining predictive SM fermion mass matrix textures that successfully describe the observed SM fermion mass and mixing pattern [12,19-21,74-103] . In the proposed model, the top and exotic quarks get tree level masses whereas the masses of the bottom, charm and strange quarks as well as the tau and muon charged lepton masses are produced from a tree level Universal Seesaw mechanism. The masses for the first generation SM charged fermions are generated from a one loop level radiative seesaw mechanism mediated by charged vector like fermions and electrically neutral scalars. The light active neutrino masses are produced from a one loop level radiative seesaw mechanism. Unlike the 3-3-1 models of Refs. [19-21,31,32,37,42,45,50] , where non renormalizable Yukawa interactions are employed for the implementation of a Froggat Nielsen mechanism to produce the current SM fermion mass and mixing pattern, after the discrete symmetries are spontaneously broken, our proposed model is a fully renormalizable theory with minimal particle content and symmetries where tree level Universal and a one-loop level radiative seesaw mechanisms are combined to explain the observed hierarchy of SM fermion masses and fermionic mixing parameters. Furthermore, unlike Refs. [19-21,31,32,37,42,45,50] our current work has an explanation for the electron and anomalous magnetic moments. In our current model, the charged vector-like leptons which mediate the tree level Universal and one-loop level radiative seesaw mechanism that generates the SM charged fermion mass hierarchy, make contributions to the measured values of the muon and electron anomalous magnetic moments, thus providing a connection of the fermion mass generation mechanism and the $g-2$ anomalies, which is not given the models of Refs. [19-21,31,32,37,42,45,50] . Our model is consistent with the low energy SM fermion flavor data and successfully accommodates the experimental values of the muon and electron magnetic dipole moments.

The content of this paper goes as follows. The model is introduced in Sect. 2. The model predictions for the muon and electron anomalous magnetic moments are discussed in Sect. 3. Section 4 is dedicated to the quark masses and mixings. Lepton masses and mixings are analyzed within the model in Sect. 5. The generation of neutrino masses is discussed in Sect. 6 . Conclusions are given in Sect. 7.

\section{The model}

We consider a renormalizable extension of the 3-3-1 model with right handed Majorana neutrinos, where the $S U(3)_{C} \times$ $S U(3)_{L} \times U(1)_{X}$ gauge symmetry is supplemented by the spontaneously broken $U(1)_{L_{g}}$ global lepton number symmetry and the $S_{3} \times Z_{2}$ discrete group, the scalar sector is enlarged by the inclusion of several gauge singlet scalars and the fermion sector is minimally augmented by the introduction of heavy electrically charged vector like fermions. Such electrically charged vector like fermions are assumed to be singlets under the $S U(3)_{L}$ gauge symmetry, thus allowing to easily comply with collider constraints as well as with the constraints arising from electroweak precision tests. The left and right handed components of such vector like fermions have the same transformation properties under the different group factors of the model thus allowing to build mass terms for these fields invariant under the $S U(3)_{C} \times S U(3)_{L} \times U(1)_{X} \times S_{3} \times Z_{2}$ group. The scalar and fermionic content with their assignments under the $S U(3)_{C} \times S U(3)_{L} \times U(1)_{X} \times S_{3} \times Z_{2}$ group are shown in Tables 1 and 2 , respectively. The dimensions of the $S U(3)_{C}$, $S U(3)_{L}$ and $S_{3}$ representations shown in Tables 1 and 2 are specified by the numbers in boldface. It is worth mentioning that the set of vector like fermions $\tilde{T}_{n}(n=1,2), B_{i}$ and $E_{i}(i=1,2,3)$ is the minimum amount of exotic fermions required to generate the tree level masses via Universal seesaw mechanism for the bottom, charm and strange quarks as well as the tau and muon as well as one loop level masses for the first generation SM charged fermions, i.e., the up, down quarks and the electron. To implement such tree level Universal and radiative seesaw mechanisms we have introduced the gauge singlet scalars $\xi_{n}(n=1,2)$ and $\varphi$. In addition, the remaining gauge singlet scalars $\sigma_{i}(i=1,2,3)$ are crucial to generate the Majorana mass terms necessary to radiatively produce the light active neutrino masses. The vector like fermions mix with the SM charged fermions lighter than the top quark thus giving rise to a tree level Universal seesaw mechanism that produces the masses for the bottom, charm and strange quarks as well as the tau and muon charged lepton masses. The first generation SM charged fermions, i.e., the up, down quarks and the electron get their masses from a one loop level radiative seesaw mechanism mediated by charged vector like fermions and electrically neutral scalars. In addition, light active neutrino masses are generated from a one loop level radiative seesaw mechanism mediated by the 
right handed Majorana neutrinos and the electrically neutral components of the $S U(3)_{L}$ scalar triplet $\chi$. The smallness of the light active neutrino masses is attributed to a small mass splitting of the $\chi_{1 R}$ and $\chi_{1 I}$ scalar fields, which originates from the trilinear term $A\left(\chi^{\dagger} \eta \sigma_{3}+h . c\right)$ of the scalar potential given in Appendix B. Thus, the trilinear coupling $A$ has to be sufficiently small to provide a natural explanation for the tiny masses of the light active neutrinos. In Sect. 6 we discuss a symmetry-based condition for technically natural smallness of the parameter $A$. Notice that the $U(1)_{L_{g}}$ global lepton number symmetry is spontaneously broken down to a residual discrete $Z_{2}^{\left(L_{g}\right)}$ by the vacuum expectation value (VEV) of the $U(1)_{L_{g}}$ charged gauge-singlet scalars $\sigma_{i}(i=1,2,3)$ having a nontrivial $U(1)_{L_{g}}$ charge, as indicated by Table 1 . The residual discrete $Z_{2}^{\left(L_{g}\right)}$ lepton number symmetry, under which the leptons are charged and the other particles are neutral, forbids interactions having an odd number of leptons, thus preventing proton decay. The massless Goldstone boson, i.e., the Majoron, arising after the spontaneous breaking of the $U(1)_{L_{g}}$ symmetry, does not cause problems in the model because it is a $S U(3)_{L}$ scalar singlet.

In addition, our model does not have fermions with exotic electric charges. Thus, the electric charge in our model is defined as follows:

$Q=T_{3}+\beta T_{8}+X=T_{3}-\frac{1}{\sqrt{3}} T_{8}+X$.

Furthermore, the lepton number has a gauge component as well as a complementary global one, as indicated by the following relation:

$L=\frac{4}{\sqrt{3}} T_{8}+L_{g}$,

being $L_{g}$ a conserved charge associated with the $U(1)_{L_{g}}$ global lepton number symmetry.

In our model the full symmetry $\mathcal{G}$ experiences the following spontaneous symmetry breaking chain:

$$
\begin{aligned}
\mathcal{G}= & S U(3)_{C} \times S U(3)_{L} \times U(1)_{X} \times U(1)_{L_{g}} \times S_{3} \times Z_{2} \stackrel{v_{x}, v_{\xi}, v_{\varphi},}{\longrightarrow} \\
& S U(3)_{C} \times S U(2)_{L} \times U(1)_{L_{g}} \stackrel{v_{\eta}, v_{\rho}}{\longrightarrow} \\
& S U(3)_{C} \times U(1)_{Q} \times U(1)_{L_{g}} \stackrel{v_{\sigma}, v_{\sigma_{3}}}{\longrightarrow} \\
& S U(3)_{C} \times U(1)_{Q},
\end{aligned}
$$

where the different symmetry breaking scales fulfill the hierarchy:

$v_{\chi} \sim v_{\xi} \sim v_{\varphi} \gg v_{\eta}, v_{\rho} \gg v_{\sigma} \sim v_{\sigma_{3}}$,

with $v_{\eta}^{2}+v_{\rho}^{2}=v^{2}, v=246 \mathrm{GeV}$. We assume that the scale $v_{\chi}$ of spontaneous $S U(3)_{L} \times U(1)_{X}$ gauge symmetry breaking is about $10 \mathrm{TeV}$ or larger in order to keep consistency with the collider constraints [104], the constraints from the
Table 1 Scalar assignments under $S U(3)_{C} \times S U(3)_{L} \times U(1)_{X} \times$ $U(1)_{L_{g}} \times S_{3} \times Z_{2}$

\begin{tabular}{llllllll}
\hline & $\chi$ & $\eta$ & $\rho$ & $\xi$ & $\varphi$ & $\sigma$ & $\sigma_{3}$ \\
\hline$S U(3)_{C}$ & $\mathbf{1}$ & $\mathbf{1}$ & $\mathbf{1}$ & $\mathbf{1}$ & $\mathbf{1}$ & $\mathbf{1}$ & $\mathbf{1}$ \\
$S U(3)_{L}$ & $\mathbf{3}$ & $\mathbf{3}$ & $\mathbf{3}$ & $\mathbf{1}$ & $\mathbf{1}$ & $\mathbf{1}$ & $\mathbf{1}$ \\
$U(1)_{X}$ & $-\frac{1}{3}$ & $-\frac{1}{3}$ & $\frac{2}{3}$ & 0 & 0 & 0 & 0 \\
$U(1)_{L_{g}}$ & $\frac{4}{3}$ & $-\frac{2}{3}$ & $-\frac{2}{3}$ & 0 & 0 & 2 & 2 \\
$S_{3}$ & $\mathbf{1}^{\prime}$ & $\mathbf{1}$ & $\mathbf{1}$ & $\mathbf{2}$ & $\mathbf{1}^{\prime}$ & $\mathbf{2}$ & $\mathbf{1}$ \\
$Z_{2}$ & -1 & 1 & 1 & -1 & -1 & 1 & 1 \\
\hline
\end{tabular}

experimental data on $K, D$ and $B$-meson mixings [105] and $B_{s, d} \rightarrow \mu^{+} \mu^{-}, B_{d} \rightarrow K^{*}(K) \mu^{+} \mu^{-}$decays [9,106-109]

In principle, the hierarchical VEV pattern (4), being unprotected by any symmetry, can be affected by large radiative corrections. The common remedy against this issue is to assume that our model is embedded into a more fundamental setup with additional symmetries protecting the hierarchy up to the Planck scale. The well-known examples of such setups are supersymmetry and warped five-dimensions. Formulation of the corresponding ultraviolet completion is beyond the scope of the present paper and will be done elsewhere. One can also be concerned about the classical stability of the scalar potential at the vacuum configuration (4). The latter must belong to the minimum of the model scalar potential shown in Appendix B. This means that the scalar mass squared matrices in the vacuum (4) are positively definite. Having at our disposal a large number of free parameters in the scalar potential (B1) it is reasonable to expect that this condition can be easily satisfied in a wide range of the model parameter space. In Sect. 3 we show that this is true for the benchmark point (17) used for the analysis of $(g-2)_{e, \mu}$.

The $S U(3)_{L}$ triplet scalars $\chi, \eta$ and $\rho$ can be expanded around the minimum as follows:

$$
\begin{aligned}
& \chi=\left(\begin{array}{c}
\frac{1}{\sqrt{2}}\left(\chi_{1 R}^{0}+i \chi_{1 I}^{0}\right) \\
\chi_{2}^{-} \\
\frac{1}{\sqrt{2}}\left(v_{\chi}+\xi_{\chi} \pm i \zeta_{\chi}\right)
\end{array}\right), \\
& \eta=\left(\begin{array}{c}
\frac{1}{\sqrt{2}}\left(v_{\eta}+\xi_{\eta} \pm i \zeta_{\eta}\right) \\
\eta_{2}^{-} \\
\frac{1}{\sqrt{2}}\left(\eta_{3 R}^{0}+i \eta_{3 I}^{0}\right)
\end{array}\right), \\
& \rho=\left(\begin{array}{c}
\rho_{1}^{+} \\
\frac{1}{\sqrt{2}}\left(v_{\rho}+\xi_{\rho} \pm i \zeta_{\rho}\right) \\
\rho_{3}^{+}
\end{array}\right),
\end{aligned}
$$

The $S U(3)_{L}$ fermionic antitriplets and triplets are

$$
\begin{aligned}
Q_{n L} & =\left(\begin{array}{c}
D_{n} \\
-U_{n} \\
J_{n}
\end{array}\right)_{L}, \quad Q_{3 L}=\left(\begin{array}{c}
U_{3} \\
D_{3} \\
T
\end{array}\right)_{L}, \quad L_{i L}=\left(\begin{array}{c}
v_{i} \\
l_{i} \\
v_{i}^{c}
\end{array}\right)_{L}, \\
n & =1,2, i=1,2,3 .
\end{aligned}
$$


Table 2 Fermion assignments under $S U(3)_{C} \times S U(3)_{L} \times U(1)_{X} \times U(1)_{L_{g}} \times S_{3} \times Z_{2}$. Here $B_{L, R}=\left(B_{1(L, R)}, B_{2(L, R)}\right), E_{L, R}=\left(E_{1(L, R)}, E_{2(L, R)}\right)$, $L_{L}=\left(L_{1(L)}, L_{2(L)}\right), N_{R}=\left(N_{1(R)}, N_{2(R)}\right), n=1,2$ and $i=1,2,3$

\begin{tabular}{lllllllllllllllllllllllll}
\hline & $Q_{L}$ & $Q_{3 L}$ & $U_{n R}$ & $U_{3 R}$ & $D_{i R}$ & $T_{R}$ & $J_{R}$ & $\widetilde{T}_{L}$ & $\widetilde{T}_{R}$ & $B_{L}$ & $B_{R}$ & $B_{3 L}$ & $B_{3 R}$ & $L_{L}$ & $L_{3 L}$ & $l_{i R}$ & $E_{L}$ & $E_{R}$ & $E_{3 L}$ & $E_{3 R}$ & $N_{R}$ & $N_{3 R}$ \\
\hline$S U(3)_{C}$ & $\mathbf{3}$ & $\mathbf{3}$ & $\mathbf{3}$ & $\mathbf{3}$ & $\mathbf{3}$ & $\mathbf{3}$ & $\mathbf{3}$ & $\mathbf{3}$ & $\mathbf{3}$ & $\mathbf{3}$ & $\mathbf{3}$ & $\mathbf{3}$ & $\mathbf{3}$ & $\mathbf{1}$ & $\mathbf{1}$ & $\mathbf{1}$ & $\mathbf{1}$ & $\mathbf{1}$ & $\mathbf{1}$ & $\mathbf{1}$ & $\mathbf{1}$ & $\mathbf{1}$ \\
$S U(3)_{L}$ & $\overline{\mathbf{3}}$ & $\mathbf{3}$ & $\mathbf{1}$ & $\mathbf{1}$ & $\mathbf{1}$ & $\mathbf{1}$ & $\mathbf{1}$ & $\mathbf{1}$ & $\mathbf{1}$ & $\mathbf{1}$ & $\mathbf{1}$ & $\mathbf{1}$ & $\mathbf{1}$ & $\mathbf{3}$ & $\mathbf{3}$ & $\mathbf{1}$ & $\mathbf{1}$ & $\mathbf{1}$ & $\mathbf{1}$ & $\mathbf{1}$ & $\mathbf{1}$ & $\mathbf{1}$ \\
$U(1)_{X}$ & 0 & $\frac{1}{3}$ & $\frac{2}{3}$ & $\frac{2}{3}$ & $-\frac{1}{3}$ & $\frac{2}{3}$ & $-\frac{1}{3}$ & $\frac{2}{3}$ & $\frac{2}{3}$ & $-\frac{1}{3}$ & $-\frac{1}{3}$ & $-\frac{1}{3}$ & $-\frac{1}{3}$ & $-\frac{1}{3}$ & $-\frac{1}{3}$ & $-\frac{1}{3}$ & -1 & -1 & -1 & -1 & 0 & 0 \\
$U(1)_{L_{g}}$ & $\frac{2}{3}$ & $-\frac{2}{3}$ & 0 & 0 & 0 & -2 & 2 & 0 & 0 & 0 & 0 & 0 & 0 & $\frac{1}{3}$ & $\frac{1}{3}$ & 1 & 1 & 1 & 1 & 1 & -1 & -1 \\
$S_{3}$ & $\mathbf{2}$ & $\mathbf{1}$ & $\mathbf{1}^{\prime}$ & $\mathbf{1}$ & $\mathbf{1}^{\prime}$ & $\mathbf{1}^{\prime}$ & $\mathbf{2}$ & $\mathbf{2}$ & $\mathbf{2}$ & $\mathbf{2}$ & $\mathbf{2}$ & $\mathbf{1}$ & $\mathbf{1}$ & $\mathbf{2}$ & $\mathbf{1}$ & $\mathbf{1}^{\prime}$ & $\mathbf{2}$ & $\mathbf{2}$ & $\mathbf{1}$ & $\mathbf{1}$ & $\mathbf{2}$ & $\mathbf{1}$ \\
$Z_{2}$ & 1 & 1 & -1 & 1 & -1 & -1 & 1 & 1 & 1 & 1 & 1 & 1 & 1 & 1 & 1 & -1 & 1 & 1 & 1 & 1 & 1 & -1 \\
\hline
\end{tabular}

where $l_{1,2,3}=e, \mu, \tau$.

With the field assignment specified in Tables 1 and 2, the following quark and lepton Yukawa terms arise:

$$
\begin{aligned}
-\mathcal{L}_{Y}^{(q)}= & y_{T} \bar{Q}_{3 L} \chi T_{R}+y_{J}\left(\bar{Q}_{L} \chi^{*} J_{R}\right)_{1}+y_{U} \bar{Q}_{3 L} \eta U_{3 R} \\
& +m_{\widetilde{T}}\left(\overline{\widetilde{T}}_{L} \widetilde{T}_{R}\right)_{\mathbf{1}}+m_{B}\left(\bar{B}_{L} B_{R}\right)_{\mathbf{1}}+m_{B_{3}} \bar{B}_{3 L} B_{3 R} \\
& +x_{T}\left(\bar{Q}_{L} \rho^{*} \widetilde{T}_{R}\right)_{\mathbf{1}}+\sum_{n=1}^{2} z_{n}^{(U)}\left(\bar{T}_{L} \xi\right)_{\mathbf{1}^{\prime}} U_{n R} \\
& +x_{B}\left(\bar{Q}_{L} \eta^{*} B_{R}\right)_{\mathbf{1}}+\sum_{j=1}^{3} z_{j}^{(D)}\left(\bar{B}_{L} \xi\right)_{\mathbf{1}^{\prime}} D_{j R} \\
-\mathcal{L}_{Y}^{(l)}= & x_{E}\left(\bar{L}_{L} \rho E_{R}\right)_{\mathbf{1}}+\sum_{j=1}^{3} z_{j}^{(l)}\left(\bar{E}_{L} \xi\right)_{\mathbf{1}^{\prime}} l_{j R} \\
& +y_{B} \bar{Q}_{3 L} \rho B_{3 R}+\sum_{j=1}^{3} x_{j}^{(D)} \bar{B}_{3 L} \varphi D_{j R}+H . c,(7) \\
& +y_{E} \bar{L}_{3 L} \rho E_{3 R}+\sum_{j=1}^{3} x_{j}^{(l)} \bar{E}_{3 L} \varphi l_{j R} \\
& +m_{E}\left(\bar{E}_{L} E_{R}\right)_{\mathbf{1}}+m_{E_{3}} \bar{E}_{3 L} E_{3 R} \\
& +x_{N}\left(\bar{L}_{L} \chi N_{R}\right)_{\mathbf{1}}+y_{N} \bar{L}_{3 L} \chi N_{3 R} \\
& +h_{1 N}\left(N_{R} \overline{N_{R}^{C}}\right)_{\mathbf{2}} \sigma+h_{2 N}\left(N_{R} \overline{N_{3 R}^{C} \sigma}\right)_{\mathbf{1}^{\prime}} \\
& +h_{3 N}\left(N_{R} \overline{N_{R}^{C}}\right)_{\mathbf{1}} \sigma_{3}+h_{4 N} N_{3 R} \overline{N_{3 R}^{C}} \sigma_{3}+H . c .
\end{aligned}
$$

We consider the following VEV configurations for the $S_{3}$ doublets:

$\langle\xi\rangle=v_{\xi}(1,0), \quad\langle\sigma\rangle=\left(v_{\sigma_{1}}, v_{\sigma_{2}}\right)$

which are consistent with the scalar potential minimization equations for a large region of parameter space $[20,90,110]$.

\section{Muon and electron anomalous magnetic moments}

The current experimental data on the anomalous dipole magnetic moments of electron and muon $a_{e, \mu}=\left(g_{e, \mu}-2\right) / 2$ show significant deviation from their SM values

$$
\begin{aligned}
\Delta a_{\mu} & =a_{\mu}^{\exp }-a_{\mu}^{\mathrm{SM}} \\
& =(26.1 \pm 8) \times 10^{-10} \\
\Delta a_{e} & =a_{e}^{\exp }-a_{e}^{\mathrm{SM}} \\
& =(-0.88 \pm 0.36) \times 10^{-12}
\end{aligned}
$$

Here we analyze predictions of our model for these observables. The leading contributions to $\Delta a_{e, \mu}$ arising in the model are shown in Fig. 1. The diagrams involve the electrically neutral physical $\mathrm{CP}$ even $H_{i}^{0}(i=1,2,3,4)$ and $\mathrm{CP}$ odd $A^{0}$ scalar as well as heavy exotic charged $E_{L, R}$ leptons. The physical CP even scalars arise from the combinations of $\xi_{\rho}$, $\xi_{1 R}, \xi_{2 R}, \varphi_{R}$ whereas the $\mathrm{CP}$ odd scalar corresponds to $\zeta_{\rho}$. By $\xi_{1 R, 2 R}$ we denote real part of the two components of the scalar $S_{3}$-doublet gauge singlet $\xi$. Similary, the real part of the scalar $S_{3}$-singlet gauge singlet $\varphi$ is denoted by $\varphi_{R}$. Analogously, $E_{1,2}$ are two components of the leptonic $S_{3}$-doublet gauge singlet $E_{L, R}$. The fields $\xi_{\rho}$ and $\zeta_{\rho}$ are contained in the $S U(3)_{L}$ scalar triplet $\rho$, which interacts with $l$, the second component of the leptonic triplet $L_{L}$. It is worth mentioning that, in view of the large amount of parametric freedom of the model scalar potential in Eq. (B1), we are restricting to a particular benchmark scenario were the $S U(3)_{L}$ scalar triplet $\rho$ and the gauge singlet scalars $\xi$ and $\varphi$ do not feature mixings with the remaining scalar fields $\eta, \sigma$ and $\sigma_{3}$. Such benchmark scenario is consistent with the decoupling limit where the $\mathrm{CP}$ even neutral component of the $S U(3)_{L}$ scalar triplet $\eta$ mostly corresponds to the $126 \mathrm{GeV}$ SM like Higgs boson. Another motivation for such benchmark scenario is the fact that the VEV of the $S U(3)_{L}$ scalar triplet $\chi$ is much larger than the VEV of the $S U(3)_{L}$ scalar triplet $\rho$, thus allowing to neglect the mixing angles between those fields since they are suppressed by the ratios of their VEVs, as follows from the method of recursive expansion of Ref. [119]. Due to the same argument, the mixing angles of the 
Fig. 1 Leading Loop Feynman diagrams contributing to the muon and electron anomalous magnetic moments. Here $E_{1,2}$, are components of the $S_{3}$ -doublet and $j=1,2,3,4$
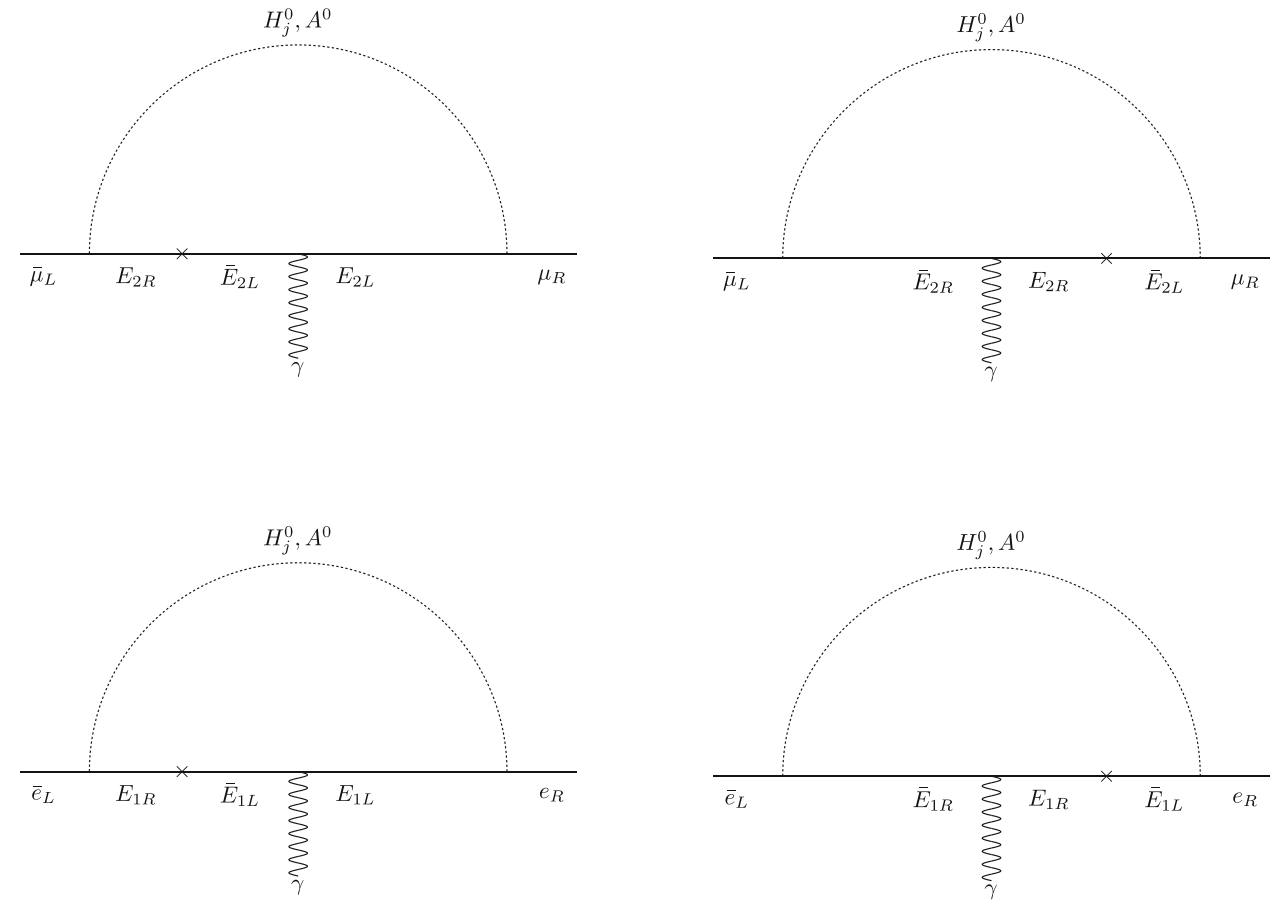

$\rho, \xi$ and $\varphi$ scalar fields with the gauge singlet scalars $\sigma$ and $\sigma_{3}$ can be neglected as well.

In this framework, the scalar potential terms contributing to the Yukawa couplings of the fermions $E_{1}$ and $E_{2}$ with the scalar fields are shown in Appendix C. Let us note the the following peculiar pattern of mixing in the scalar sector. The fields $\rho, \xi$ and $\varphi$ do not mix with $\eta, \sigma$ and $\sigma_{3}$, while $\varphi$ mix with $\zeta_{\rho}$ through the the complex parameter $\kappa$ in the scalar potential (B1). In view of this we find that the scalar mass matrix in the basis $\xi_{\rho}, \xi_{1 R}, \xi_{2 R}, \varphi$, and $\zeta_{\rho}$ has the form:

$\mathbf{M}^{2}=\left(\begin{array}{ccccc}m_{11}^{2} & m_{12}^{2} & 0 & m_{14}^{2} & 0 \\ m_{21}^{2} & m_{22}^{2} & m_{23}^{2} & m_{24}^{2} & 0 \\ 0 & m_{32} & m_{33} & m_{34} & 0 \\ m_{41}^{2} & m_{42}^{2} & m_{43}^{2} & m_{44}^{2} & m_{45}^{2} \\ 0 & 0 & 0 & m_{54}^{2} & m_{55}^{2}\end{array}\right)$

with the matrix elements $m_{i j}^{2}$ given in Appendix C. Once the basis is changed by a rotation matrix $R$, the physical scalar field masses $m_{H_{1}^{0}}, m_{H_{2}^{0}}, m_{H_{3}^{0}}, m_{H_{5}^{0}}$ and $m_{H_{A}^{0}}$ can be found numerically.

Thus, in our model the muon and electron anomalous magnetic moments are given by:

$\Delta a_{\mu, e}=\sum_{i=1}^{4} \sum_{\Phi=H_{i}^{0}, A^{0}} \Delta a_{\mu, e}(\Phi)$,

The analytical form for the neutral scalar contribution at one loop to $\Delta a_{\mu, e}$ can be found in [22,120-122]. Using these results we write the contributions of the neutral scalars $\Phi=$
$H^{0}, A^{0}$ as follows:

$$
\begin{aligned}
\Delta a_{\mu}= & w_{\mu}^{2} \frac{m_{\mu}^{2}}{8 \pi^{2}}\left\{\sum_{i=1}^{4}\left(R^{T}\right)_{1 i}\left(R^{T}\right)_{2 i} \frac{G_{S}^{(l)}\left(m_{E_{2}}, m_{H_{i}^{0}}\right)}{m_{H_{i}^{0}}^{2}}\right. \\
& \left.+\left(R^{T}\right)_{55}\left(R^{T}\right)_{25} \frac{G_{P}^{(l)}\left(m_{E_{2}}, m_{A^{0}}\right)}{m_{A^{0}}^{2}}\right\} \\
\Delta a_{e}= & w_{e}^{2} \frac{m_{\mu}^{2}}{8 \pi^{2}}\left\{\sum_{i=1}^{4}\left(R^{T}\right)_{1 i}\left(R^{T}\right)_{3 i} \frac{G_{S}^{(l)}\left(m_{E_{1}}, m_{H_{i}^{0}}\right)}{m_{H_{i}^{0}}^{2}}\right. \\
& \left.+\left(R^{T}\right)_{55}\left(R^{T}\right)_{35} \frac{G_{P}^{(l)}\left(m_{E_{1}}, m_{A^{0}}\right)}{m_{A^{0}}^{2}}\right\}
\end{aligned}
$$

where the loop function is given by:

$$
\begin{aligned}
& G_{S, P}^{(l)}\left(m_{E}, m_{\Phi}\right) \\
& \quad=\int_{0}^{1} d x \frac{x^{2}\left(1-x \pm \epsilon_{l E}\right)}{(1-x)\left(1-x \lambda_{l \Phi}^{2}\right)+x \epsilon_{l E}^{2} \lambda_{l \Phi}^{2}}, \quad \Phi=H^{0}, A^{0}
\end{aligned}
$$

with $l=e, \mu$ and $\lambda_{l \Phi}=m_{l} / m_{\Phi}, \epsilon_{e E}=m_{E_{1}} / m_{e}, \epsilon_{\mu E}=$ $m_{E_{2}} / m_{\mu}$. Besides that, the plus and minus signs for the loop function $G_{S, P}(\Phi)$ of Eq. (16) stands for the scalar (CP-even) and pseudoscalar (CP-odd) contributions, respectively. The quantities $w_{l}(l=e, \mu)$ are the Yukawa couplings for the interaction $w_{l} \bar{E} l \Phi$.

The experimental values of the muon and electron anomalous magnetic moments shown in Eqs. (10) and (11) can be successfully reproduced at $2 \sigma$ level for the following benchmark point: 


$$
\begin{aligned}
& v_{\eta}=v_{\rho} \approx 174 \mathrm{GeV} \quad v_{\chi} \approx 2851 \mathrm{GeV} \quad v_{\xi} \approx 1414 \mathrm{GeV} \\
& v_{\phi} \approx 6992 \mathrm{GeV} \quad \kappa_{r} \approx-0.630 \quad \kappa_{i} \approx-0.614 \\
& \lambda_{2}=\lambda_{10} \approx 7.251 \quad \lambda_{12}=\lambda_{13} \approx 0.310 \\
& \lambda_{18}=\lambda_{34} \approx-0.264 \quad \lambda_{35}=\lambda_{38} \approx-0.229
\end{aligned}
$$

The scalar and charged exotic leptons masses along with the Yukawa couplings are

$$
\begin{aligned}
& m_{H_{1}^{0}} \approx 5786 \mathrm{GeV} \quad m_{H_{2}^{0}} \approx 5338 \mathrm{GeV} \quad m_{H_{3}^{0}} \approx 2750 \mathrm{GeV} \\
& m_{H_{4}^{0}} \approx 2498 \mathrm{GeV} \quad m_{A^{0}} \approx 1100 \mathrm{GeV} \quad m_{E_{1}} \approx 611 \mathrm{GeV} \\
& m_{E_{2}} \approx 1368 \mathrm{GeV} \quad w_{\mu} \approx 0.228 \quad w_{e} \approx 1.719
\end{aligned}
$$

Note that this benchmark point locates in the domain of the model parameter space corresponding to the minimum of the scalar potential due to the fact that all the scalar masses are real (see also Appendix C). In this benchmark point the muon and electron $(g-2)$-experimental anomalies have the values

$$
\begin{aligned}
\Delta a_{\mu} & =2.68714 \times 10^{-9} \\
\Delta a_{e} & =-8.64531 \times 10^{-13}
\end{aligned}
$$

The opposite signs of these quantities is due to the pseudo scalar $A^{0}$ contributions to the loops in Fig. 1 leading to the minus sign in the term $-\epsilon_{l E}$ of the loop function (16). Note that $E_{2}$ and $E_{1}$ contribute separately to the muon and electron $(g-2)$, respectively, without any cross-contributions. Thus, selecting appropriate values for the exotic lepton masses $m_{E_{1,2}}$ we can accommodate the experimental sign difference (19), (20). The fact that $m_{e} \ll m_{\mu}$ makes the required sign difference valid in a wide range of the model parameter space. To show this, let us vary the model parameters within $15 \%$ around the benchmark point (17) and the charged exotic lepton masses in a range from 200 to $1200 \mathrm{GeV}$. The resulting $\Delta a_{\mu, e}-m_{E_{2,1}}$ scatter plots are shown in Fig. 2. As can be seen, the model indeed can explain the experimental values of muon and electron anomalous magnetic moments simultaneously in a wide range of its parameter space.

\section{Quark masses and mixings}

From the quark Yukawa interactions in Eq. (7), we find that the up-type mass matrix in the basis $\left(\bar{u}_{1 L}, \bar{u}_{2 L}, \bar{u}_{3 L}, \bar{T}_{L}\right.$, $\left.\widetilde{T}_{1 L}, \widetilde{T}_{2 L}\right)$ versus $\left(u_{1 R}, u_{2 R}, u_{3 R}, T_{R}, \widetilde{T}_{1 R}, \widetilde{T}_{2 R}\right)$ is given by:

$$
M_{U}=\left(\begin{array}{cccc}
\Delta_{U} & 0_{2 \times 1} & 0_{2 \times 1} & A_{U} \\
0_{1 \times 2} & m_{t} & 0 & 0_{1 \times 2} \\
0_{1 \times 2} & 0 & m_{T} & 0_{1 \times 2} \\
B_{U} & 0_{2 \times 1} & 0_{2 \times 1} & \widetilde{M}_{T}
\end{array}\right),
$$

$$
\begin{aligned}
A_{U}= & x_{T}\left(\begin{array}{ll}
1 & 0 \\
0 & 1
\end{array}\right) \frac{v_{\rho}}{\sqrt{2}}, \quad B_{U}=\left(\begin{array}{cc}
0 & 0 \\
z_{1}^{(U)} & z_{2}^{(U)}
\end{array}\right) v_{\xi}, \\
m_{t}= & y_{U} \frac{v_{\eta}}{\sqrt{2}}=a_{3}^{(U)} \frac{v}{\sqrt{2}}, \quad \tilde{M}_{T}=m_{\widetilde{T}}\left(\begin{array}{ll}
1 & 0 \\
0 & 1
\end{array}\right), \\
\Delta_{U}= & \left(\begin{array}{ll}
\varepsilon_{11}^{(U)} & \varepsilon_{12}^{(U)} \\
\varepsilon_{21}^{(U)} & \varepsilon_{22}^{(U)}
\end{array}\right) \frac{v_{\rho}}{\sqrt{2}}, \\
\varepsilon_{1 n}^{(U)}= & \frac{1}{16 \pi^{2}} \frac{\lambda_{\rho^{\dagger} \rho \xi^{2}} \lambda_{\xi^{3} \varphi} x_{T} z_{n}^{(U)} v_{\varphi} v_{\xi}^{2}}{m_{T} m_{\xi_{2}}^{2}}\left[C_{0}\left(\frac{m_{\xi_{\eta}}}{m_{B}}, \frac{m_{\mathrm{Re} \xi_{2}}}{m_{B}}\right)\right. \\
& \left.-C_{0}\left(\frac{m_{\zeta_{\eta}}}{m_{B}}, \frac{m_{\operatorname{Im} \xi_{2}}}{m_{B}}\right)\right], \quad n=1,2, \\
\varepsilon_{2 n}^{(U)}= & \frac{1}{16 \pi^{2}} \frac{\lambda_{\rho^{\dagger} \rho \xi^{2}} x_{T} z_{n}^{(U)} v_{\xi}}{m_{T}}\left[C_{0}\left(\frac{m_{\xi_{\eta}}}{m_{B}}, \frac{m_{\mathrm{Re} \xi_{2}}}{m_{B}}\right)\right. \\
& \left.-C_{0}\left(\frac{m_{\zeta_{\eta}}}{m_{B}}, \frac{m_{\mathrm{Im} \xi_{2}}}{m_{B}}\right)\right],
\end{aligned}
$$

whereas the down type quark mass matrix written in the basis $\left(\bar{d}_{1 L}, \bar{d}_{2 L}, \bar{d}_{3 L}, \bar{J}_{1 L}, \bar{J}_{2 L}, \bar{B}_{1 L}, \bar{B}_{2 L}, \bar{B}_{3 L}\right)-\left(d_{1 R}, d_{2 R}\right.$, $\left.d_{3 R}, J_{1 R}, J_{2 R}, B_{1 R}, B_{2 R}, B_{3 R}\right)$ takes the form:

$$
\begin{aligned}
& M_{D}=\left(\begin{array}{ccc}
\Delta_{D} & 0_{3 \times 2} & A_{D} \\
0_{2 \times 3} & M_{J} & 0_{2 \times 3} \\
B_{D} & 0_{3 \times 2} & M_{B}
\end{array}\right), A_{D}=\left(\begin{array}{ccc}
x_{B} \frac{v_{\eta}}{\sqrt{2}} & 0 & 0 \\
0 & x_{B} \frac{v_{\eta}}{\sqrt{2}} & 0 \\
0 & 0 & y_{B} \frac{v_{\rho}}{\sqrt{2}}
\end{array}\right) \text {, } \\
& B_{D}=\left(\begin{array}{cccc}
0 & 0 & 0 \\
z_{1}^{(D)} v_{\xi} & z_{2}^{(D)} v_{\xi} & z_{3}^{(D)} v_{\xi} \\
x_{1}^{(D)} v_{\varphi} & x_{2}^{(D)} v_{\varphi} & x_{3}^{(D)} v_{\varphi}
\end{array}\right), \\
& M_{J}=y^{(J)} \frac{v_{\chi}}{\sqrt{2}}\left(\begin{array}{ll}
1 & 0 \\
0 & 1
\end{array}\right), \quad M_{B}=\left(\begin{array}{ccc}
m_{B} & 0 & 0 \\
0 & m_{B} & 0 \\
0 & 0 & m_{B_{3}}
\end{array}\right), \\
& \Delta_{D}=\left(\begin{array}{lll}
\varepsilon_{11}^{(D)} & \varepsilon_{12}^{(D)} & \varepsilon_{13}^{(D)} \\
\varepsilon_{21}^{(D)} & \varepsilon_{22}^{(D)} & \varepsilon_{23}^{(D)} \\
\varepsilon_{31}^{(D)} & \varepsilon_{32}^{(D)} & \varepsilon_{33}^{(D)}
\end{array}\right) \frac{v_{\rho}}{\sqrt{2}} \\
& \varepsilon_{1 i}^{(D)}=\frac{1}{16 \pi^{2}} \frac{\lambda_{\rho^{\dagger} \rho \xi^{2}} \lambda_{\xi^{3} \varphi} x_{B} z_{i}^{(D)} v_{\varphi} v_{\xi}^{2} v_{\eta}}{m_{B} m_{\xi_{2}}^{2} v_{\rho}} C_{0}\left(\frac{m_{\xi_{\eta}}}{m_{B}}, \frac{m_{\operatorname{Re} \xi_{2}}}{m_{B}}\right) \text {, } \\
& \varepsilon_{2 i}^{(D)}=\frac{1}{16 \pi^{2}} \frac{\lambda \rho^{\dagger} \rho \xi^{2} x_{B} z_{i}^{(D)} v_{\xi} v_{\eta}}{m_{B} v_{\rho}} C_{0}\left(\frac{m_{\xi_{\eta}}}{m_{B}}, \frac{m_{\mathrm{Re} \xi_{2}}}{m_{B}}\right), \\
& \varepsilon_{3 i}^{(D)}=\frac{1}{16 \pi^{2}} \frac{\lambda_{\rho^{\dagger} \rho \varphi^{2}} x_{B} z_{i}^{(D)} v_{\varphi}}{m_{B}} C_{0}\left(\frac{m_{\xi_{\rho}}}{m_{B}}, \frac{m_{\operatorname{Re} \varphi}}{m_{B}}\right), \\
& i=1,2,3 \text {, }
\end{aligned}
$$

where as seen from Eqs. (21) and (22), the $\Delta_{U}$ and $\Delta_{D}$ submatrices are generated at one loop level. The one loop level Feynman diagrams generating the $\Delta_{U}$ and $\Delta_{D}$ submatrices are shown in Fig. 3. In addition, the following function has been introduced: 


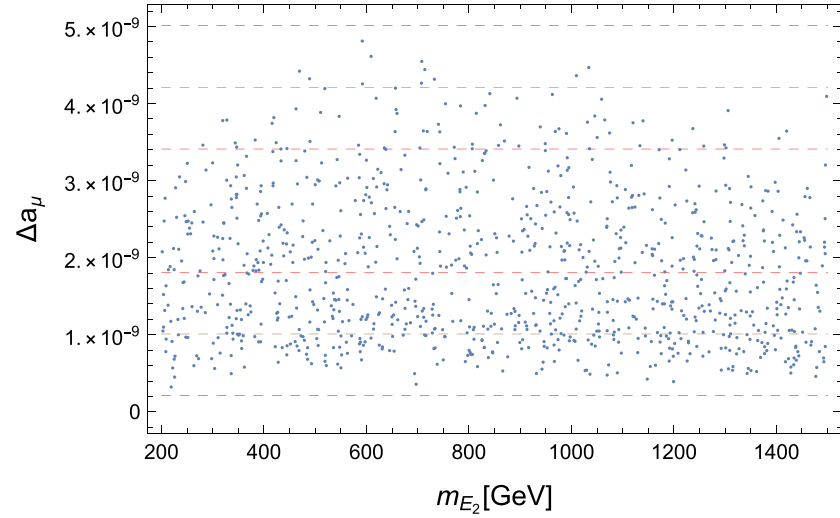

(a) Correlation plot between the mass of the exotic fermion $E_{2}$ and the value of $\Delta a_{\mu}$.

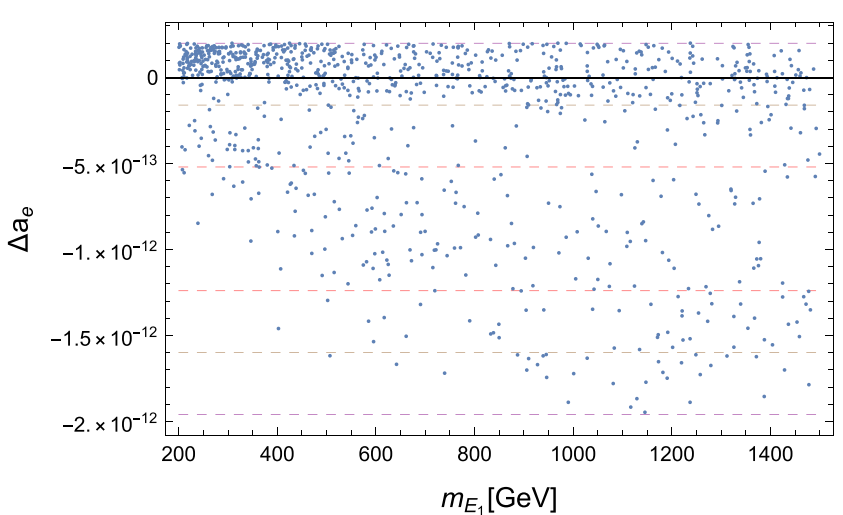

(b) Correlation plot between the mass of the exotic fermion $E_{1}$ and the value of $\Delta a_{e}$

Fig. 2 Correlation plots of the $\Delta a_{\mu, e}$ and the mass of the exotic fermion $m_{E_{2,1}}$ respectively at $1 \sigma$ (red), $2 \sigma$ (brown) and $3 \sigma$ (purple)

$$
\begin{aligned}
& C_{0}\left(\widehat{m}_{1}, \widehat{m}_{2}\right) \\
& =\frac{1}{\left(1-\widehat{m}_{1}^{2}\right)\left(1-\widehat{m}_{2}^{2}\right)\left(\widehat{m}_{1}^{2}-\widehat{m}_{2}^{2}\right)}\left\{\widehat{m}_{1}^{2} \widehat{m}_{2}^{2} \ln \left(\frac{\widehat{m}_{1}^{2}}{\widehat{m}_{2}^{2}}\right)\right. \\
& \left.\quad-\widehat{m}_{1}^{2} \ln \widehat{m}_{1}^{2}+\widehat{m}_{2}^{2} \ln \widehat{m}_{2}^{2}\right\} .
\end{aligned}
$$

As seen from Eqs. (21) and (22), the exotic heavy vector like quarks mix with the SM quarks lighter than top quark. The masses of these exotic quarks are assumed to be much larger than the $S U(3)_{L} \times U(1)_{X}$ symmetry breaking scale. As a result, charm, bottom and strange quarks acquire their masses from the tree-level Universal seesaw mechanism, while the masses of the up and down quarks are generated by the one-loop radiative seesaw mechanism. Thus, for the SM quarks we obtain the following mass matrices:

$$
\begin{aligned}
\tilde{M}_{U}= & \left(\begin{array}{ccc}
\Delta_{U}+A_{U} M_{\widetilde{T}}^{-1} B_{U} & 0_{2 \times 1} \\
0_{1 \times 2} & m_{t}
\end{array}\right) \\
& =\left(\begin{array}{ccc}
\varepsilon_{11}^{(U)} \frac{v_{\rho}}{\sqrt{2}} & \varepsilon_{12}^{(U)} \frac{v_{\rho}}{\sqrt{2}} & 0 \\
\varepsilon_{21}^{(U)} \frac{v_{\rho}}{\sqrt{2}}+\frac{x_{T} z_{1}^{(U)} v_{\xi} v_{\rho}}{\sqrt{2} m_{\tilde{T}}} \varepsilon_{22}^{(U)} \frac{v_{\rho}}{\sqrt{2}}+\frac{x_{T} z_{2}^{(U)} v_{\xi} v_{\rho}}{\sqrt{2} m_{\widetilde{T}}} & 0 \\
0 & 0 & m_{t}
\end{array}\right), \\
\tilde{M}_{D} & =\Delta_{D}+A_{D} M_{B}^{-1} B_{D} \\
& =\left(\begin{array}{cccc}
\varepsilon_{11}^{(D)} \frac{v_{\rho}}{\sqrt{2}} & \varepsilon_{12}^{(D)} \frac{v_{\rho}}{\sqrt{2}} & \varepsilon_{13}^{(D)} \frac{v_{\rho}}{\sqrt{2}} \\
\varepsilon_{21}^{(D)} \frac{v_{\rho}}{\sqrt{2}}+x_{B} z_{1}^{(D)} \frac{v_{\xi} v_{\eta}}{\sqrt{2} m_{B}} & \varepsilon_{22}^{(D)} \frac{v_{\rho}}{\sqrt{2}}+x_{B} z_{2}^{(D)} \frac{v_{\xi} v_{\eta}}{\sqrt{2} m_{B}} & \varepsilon_{23}^{(D)} \frac{v_{\rho}}{\sqrt{2}}+x_{B} z_{3}^{(D)} \frac{v_{\xi} v_{\eta}}{\sqrt{2} m_{B}} \\
\varepsilon_{31}^{(D)} \frac{v_{\rho}}{\sqrt{2}}+y_{B} x_{1}^{(D)} \frac{v_{\varphi} v_{\rho}}{\sqrt{2} m_{B 3}} & \varepsilon_{32}^{(D)} \frac{v_{\rho}}{\sqrt{2}}+y_{B} x_{2}^{(D)} \frac{v_{\varphi} v_{\rho}}{\sqrt{2} m_{B 3}} \varepsilon_{33}^{(D)} \frac{v_{\rho}}{\sqrt{2}}+y_{B} x_{3}^{(D)} \frac{v_{\varphi} v_{\rho}}{\sqrt{2} m_{B_{3}}}
\end{array}\right) .
\end{aligned}
$$

These mass matrices contain several model parameters. While free, they still satisfy certain conditions in our model. In fact, vev's $v_{\xi, \eta, \rho}$ obey the inequality (4) expressing the hierarchy of symmetry breaking in our model. The $\varepsilon_{i j}^{U, D}$ parameters, defined in Eqs. (21) and (22), contain typical loop suppression and specific dependence on the vev's, exotic masses, the Yukawas and a quartic coupling. We require the latter to satisfy the perturbativity condition. With this in mind we can speak about natural values of the matrix elements corresponding to the values of the model parameters in a range not involving an ad hoc hierarchy of the dimensionless couplings. Let us show that within this natural range the model accommodates the observable values of the SM quark masses and mixings. To this end we consider a particular natural benchmark scenario consistent with the abovementioned conditions. We choose:

$$
\begin{aligned}
v_{\xi} & =\lambda^{4} \frac{v m_{\widetilde{T}}}{v_{\rho}}=\lambda^{5} \frac{v m_{B}}{v_{\eta}}, \quad v_{\varphi}=\lambda^{3} \frac{v m_{B_{3}}}{v_{\rho}}, \\
\varepsilon_{n m}^{(U)} & =b_{n m}^{(U)} \lambda^{8} \frac{v}{v_{\rho}},
\end{aligned}
$$

$\varepsilon_{i j}^{(D)}=b_{i j}^{(D)} \lambda^{7} \frac{v}{v_{\rho}}, \quad i, j=1,2,3 ; n, m=1,2$,

where $v=\sqrt{v_{\rho}^{2}+v_{\eta}^{2}}=246 \mathrm{GeV}$ is the electroweak symmetry breaking scale. We use the Wolfenstein parameter $\lambda=0.225$ for characterization of the hierarchy between 
Fig. 3 One-loop Feynman diagrams contributing to the entries of the SM quark mass matrices. Here, $n=1,2$ and $i=1,2,3$
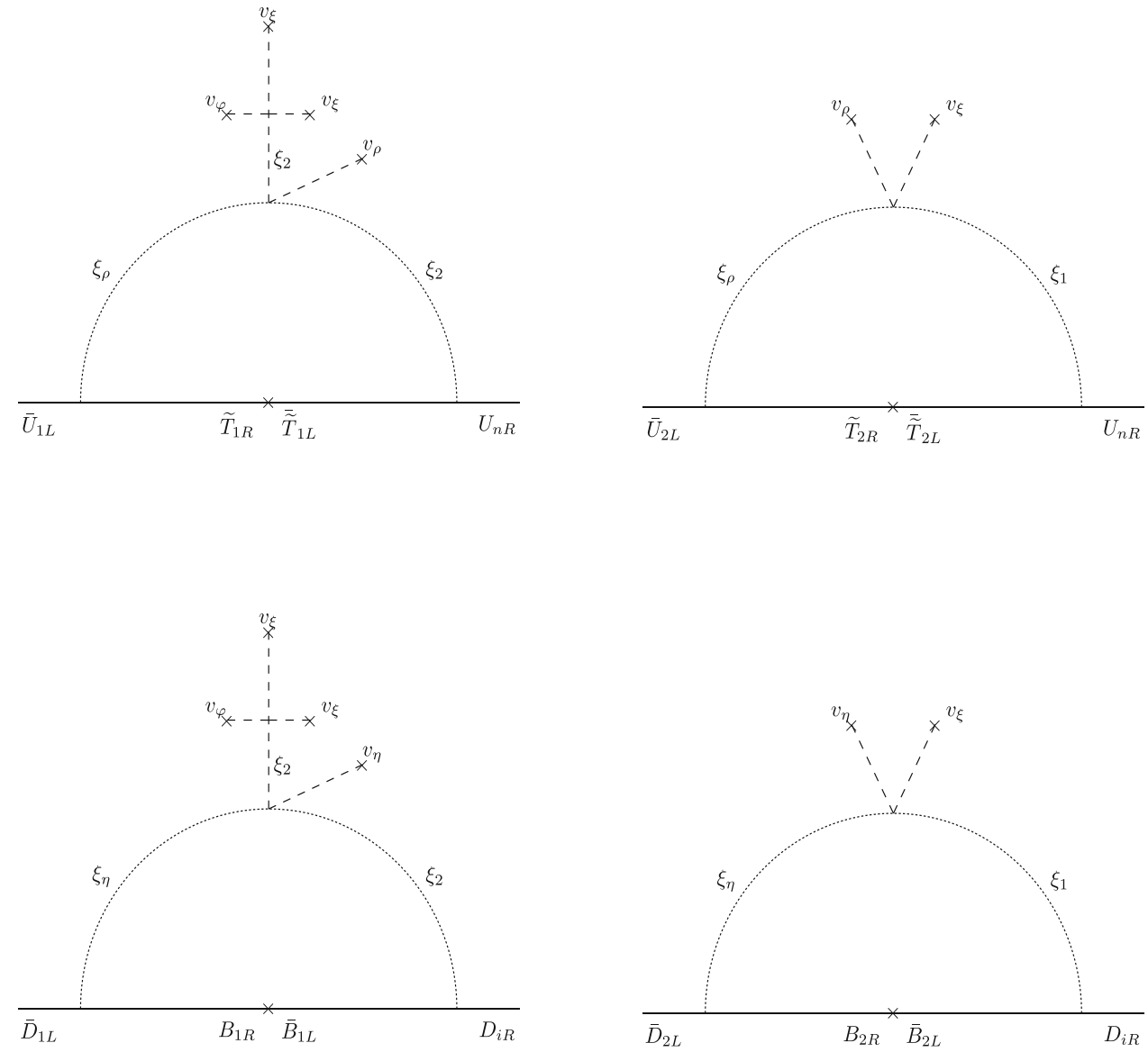

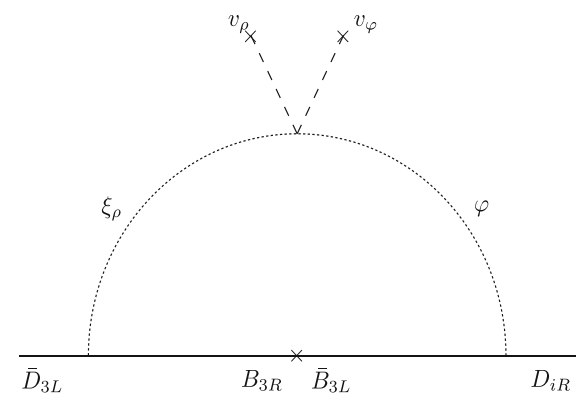

the parameters defining mass matrix elements. As discussed above, we consider the hierarchy, which stems from the model structure rather than from strong tuning of the dimensionless couplings. Then the coefficients $b_{n m}^{(U)}$ and $b_{i j}^{(D)}$, constructed from the Yukawa and quartic couplings, are $\mathcal{O}(1)$ -numbers. In the scenario (26) the exotic quarks $\tilde{T}$ and $B$ are heavier than the scale of the first stage of the symmetry breaking (3). As we mentioned earlier, these exotic quarks must be very heavy for the Universal Seesaw mechanism to operate in our model.

Thus, in the benchmark scenario (26) the SM quark mass matrices take theform:

$$
\begin{aligned}
\tilde{M}_{U} & =\left(\begin{array}{ccc}
b_{11}^{(U)} \lambda^{8} & b_{12}^{(U)} \lambda^{8} & 0 \\
b_{21}^{(U)} \lambda^{7}+a_{21}^{(U)} \lambda^{4} & b_{22}^{(U)} \lambda^{7}+a_{22}^{(U)} \lambda^{4} & 0 \\
0 & 0 & m_{t}
\end{array}\right) \frac{v}{\sqrt{2}} \\
& =\left(\begin{array}{ccc}
b_{11}^{(U)} \lambda^{8} & b_{12}^{(U)} \lambda^{8} & 0 \\
c_{21}^{(U)} \lambda^{4} & c_{22}^{(U)} \lambda^{4} & 0 \\
0 & 0 & m_{t}
\end{array}\right) \frac{v}{\sqrt{2}}, \\
\tilde{M}_{D} & =\left(\begin{array}{ccc}
b_{11}^{(D)} \lambda^{7} & b_{12}^{(D)} \lambda^{7} & b_{13}^{(D)} \lambda^{7} \\
b_{21}^{(D)} \lambda^{7}+a_{21}^{(D)} \lambda^{5} & b_{22}^{(D)} \lambda^{7}+a_{22}^{(D)} \lambda^{5} & b_{23}^{(D)} \lambda^{7}+a_{23}^{(D)} \lambda^{5} \\
b_{31}^{(D)} \lambda^{7}+a_{31}^{(D)} \lambda^{3} & b_{32}^{(D)} \lambda^{7}+a_{32}^{(D)} \lambda^{3} & b_{33}^{(D)} \lambda^{7}+a_{33}^{(D)} \lambda^{3}
\end{array}\right) \frac{v}{\sqrt{2}}
\end{aligned}
$$


Table 3 Experimental $M_{Z}$-scale values of the quark masses [123,124] and CKM parameters [125]

\begin{tabular}{ll}
\hline Observable & Experimental value \\
\hline$m_{u}(\mathrm{MeV})$ & $1.45_{-0.45}^{+0.56}$ \\
$m_{c}(\mathrm{MeV})$ & $635 \pm 86$ \\
$m_{t}(\mathrm{GeV})$ & $172.1 \pm 0.6 \pm 0.9$ \\
$m_{d}(\mathrm{MeV})$ & $2.9_{-0.4}^{+0.5}$ \\
$m_{S}(\mathrm{MeV})$ & $57.7_{-15.7}^{+16.8}$ \\
$m_{b}(\mathrm{GeV})$ & $2.82_{-0.04}^{+0.09}$ \\
$\sin \theta_{12}^{(q)}$ & 0.225 \\
$\sin \theta_{23}^{(q)}$ & 0.0421 \\
$\sin \theta_{13}^{(q)}$ & 0.00365 \\
$J$ & $(3.18 \pm 0.15) \times 10^{-5}$ \\
\hline
\end{tabular}

$$
=\left(\begin{array}{llll}
b_{11}^{(D)} \lambda^{7} & b_{12}^{(D)} \lambda^{7} & b_{13}^{(D)} \lambda^{7} \\
c_{21}^{(D)} \lambda^{5} & c_{22}^{(D)} \lambda^{5} & c_{23}^{(D)} \lambda^{5} \\
c_{31}^{(b)} \lambda^{3} & c_{32}^{(D)} \lambda^{3} & c_{33}^{(D)} \lambda^{3}
\end{array}\right) \frac{v}{\sqrt{2}},
$$

where

$$
\begin{array}{ll}
a_{21}^{(U)}=x_{T} z_{1}^{(U)}, & a_{22}^{(U)}=x_{T} z_{2}^{(U)}, \\
a_{2 i}^{(D)}=x_{B} z_{i}^{(D)}, & a_{3 i}^{(D)}=y_{B} x_{i}^{(D)},
\end{array}
$$

The model has 13 dimensionless parameters in the quark sector. This allows us to reproduce precisely the central experimental values of 10 quark observables, shown in Table 3. The corresponding values of the model parameters are:

$b_{11}^{(U)}=c_{21}^{(U)}=1$,

$$
b_{12}^{(U)} \simeq 2.773
$$

$b_{22}^{(U)} \simeq 1.001$,

$$
a_{3}^{(U)} \simeq 0.989
$$

$b_{11}^{(D)} \simeq-1.335+0.929 i, b_{12}^{(D)} \simeq 1.217+1.314 i$,

$b_{13}^{(D)} \simeq 2.112-0.929 i$,

$c_{21}^{(D)} \simeq-0.869$,

$c_{22}^{(D)} \simeq-0.438$,

$c_{13}^{(D)} \simeq 0.860$,

$c_{31}^{(D)} \simeq-0.707$,

$c_{32}^{(D)} \simeq-1.001$,

$c_{33}^{(D)} \simeq 0.707$.

An important point for us is that all these values are of the order of one. As we previously discussed, this means that the hierarchy of the quark masses and mixings originate in our model from its internal structure - symmetries and field content - without the need for strong tuning the dimensionless couplings.

\section{Charged lepton masses and mixings}

From the charged lepton Yukawa interactions in Eq. (8) we find the charged lepton mass matrix $M_{l}$ in the basis $\left(\bar{l}_{1 L}, \bar{l}_{2 L}, \bar{l}_{3 L}, \bar{E}_{1 L}, \bar{E}_{2 L}, \bar{E}_{3 L}\right)$ versus $\left(l_{1 R}, l_{2 R}, l_{3 R}, E_{1 R}\right.$, $\left.E_{2 R}, E_{3 R}\right)$ given by:

$$
\begin{aligned}
& M_{l}=\left(\begin{array}{cc}
\Delta_{l} & A_{l} \\
B_{l} & \widetilde{M}_{E}
\end{array}\right), \quad \Delta_{l}=\left(\begin{array}{ccc}
\varepsilon_{11}^{(l)} & \varepsilon_{12}^{(l)} & \varepsilon_{13}^{(l)} \\
\varepsilon_{21}^{(l)} & \varepsilon_{22}^{(l)} & \varepsilon_{23}^{(l)} \\
\varepsilon_{31}^{(l)} & \varepsilon_{32}^{(l)} & \varepsilon_{33}^{(l)}
\end{array}\right) \frac{v_{\rho}}{\sqrt{2}}, \\
& A_{l}=\left(\begin{array}{ccc}
x_{E} & 0 & 0 \\
0 & x_{E} & 0 \\
0 & 0 & y_{E}
\end{array}\right) \frac{v_{\rho}}{\sqrt{2}}, \\
& B_{l}=\left(\begin{array}{ccc}
0 & 0 & 0 \\
-z_{1}^{(l)} v_{\xi} & -z_{2}^{(l)} v_{\xi} & -z_{3}^{(l)} v_{\xi} \\
x_{1}^{(l)} v_{\varphi} & x_{2}^{(l)} v_{\varphi} & x_{3}^{(l)} v_{\varphi}
\end{array}\right), \\
& \tilde{M}_{E}=\left(\begin{array}{ccc}
m_{E} & 0 & 0 \\
0 & m_{E} & 0 \\
0 & 0 & m_{E_{3}}
\end{array}\right) \text {, } \\
& \varepsilon_{1 i}^{(l)}=\frac{1}{16 \pi^{2}} \frac{\lambda \rho_{\rho^{\dagger} \rho \xi^{2}} \lambda_{\xi^{3} \varphi} x_{E} z_{i}^{(l)} v_{\varphi} v_{\xi}^{2}}{m_{E} m_{\xi_{2}}^{2}}\left[C_{0}\left(\frac{m_{\xi_{\rho}}}{m_{E}}, \frac{m_{\mathrm{Re} \xi_{2}}}{m_{E}}\right)\right. \\
& \left.-C_{0}\left(\frac{m_{\zeta_{\rho}}}{m_{E}}, \frac{m_{\operatorname{Im} \xi_{2}}}{m_{E}}\right)\right],
\end{aligned}
$$

$$
\begin{aligned}
\varepsilon_{2 i}^{(l)}= & \frac{1}{16 \pi^{2}} \frac{\lambda \rho_{\rho^{\dagger} \rho \xi^{2}} x_{E} z_{i}^{(l)} v_{\xi}}{m_{E}}\left[C_{0}\left(\frac{m_{\xi_{\rho}}}{m_{E}}, \frac{m_{\mathrm{Re} \xi_{2}}}{m_{E}}\right)\right. \\
& \left.-C_{0}\left(\frac{m_{\zeta_{\rho}}}{m_{E}}, \frac{m_{\mathrm{Im} \xi_{2}}}{m_{E}}\right)\right],
\end{aligned}
$$

$$
\begin{aligned}
\varepsilon_{3 i}^{(l)}= & \frac{1}{16 \pi^{2}} \frac{\lambda_{\rho^{\dagger} \rho \varphi^{2}} x_{E} z_{i}^{(E)} v_{\varphi}}{m_{E}}\left[C_{0}\left(\frac{m_{\xi_{\rho}}}{m_{E}}, \frac{m_{\operatorname{Re} \varphi}}{m_{E}}\right)\right. \\
& \left.-C_{0}\left(\frac{m_{\zeta_{\rho}}}{m_{E}}, \frac{m_{\operatorname{Im} \varphi}}{m_{E}}\right)\right], \quad i=1,2,3 .
\end{aligned}
$$

where as seen from Eq. (31), the $\Delta_{l}$ submatrix is generated at one loop level according to the Feynman diagrams shown in Fig. 4.

As follows from Eq. (31), the very heavy vector like charged leptons mix with the SM charged leptons. The former are assumed to have masses much larger than the $S U(3)_{L} \times U(1)_{X}$ symmetry breaking scale. Therefore, analogously to the quark sector, the tau and muon masses are generated by the tree level Universal seesaw mechanism, while the electron mass arises from the one loop level radiative seesaw mechanism. Consequently, SM charged lepton mass matrix takes theform 
Fig. 4 One-loop Feynman diagrams contributing to the entries of the SM charged lepton mass matrix. Here $i=1,2,3$
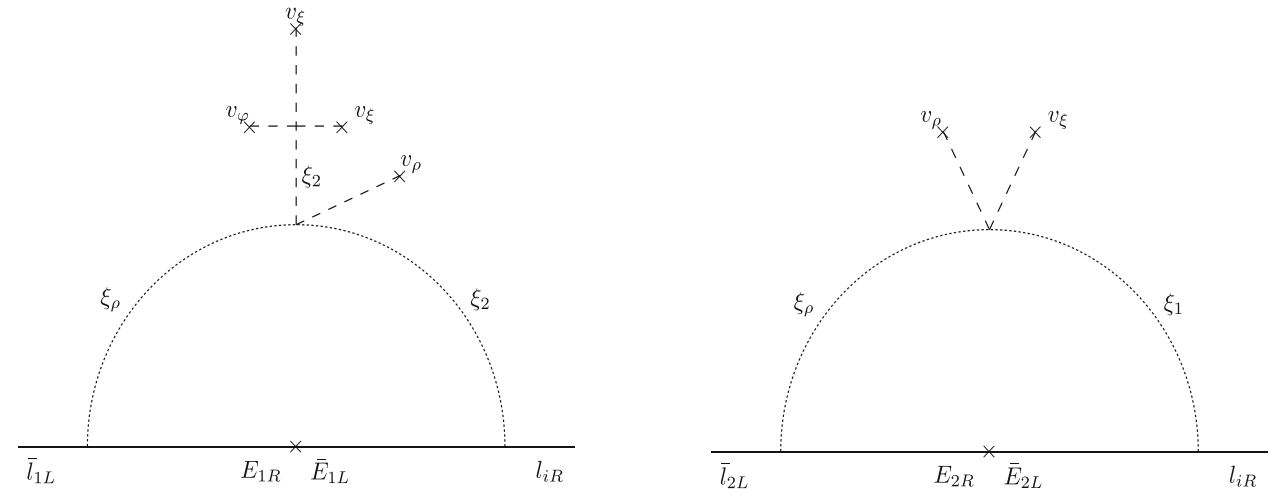

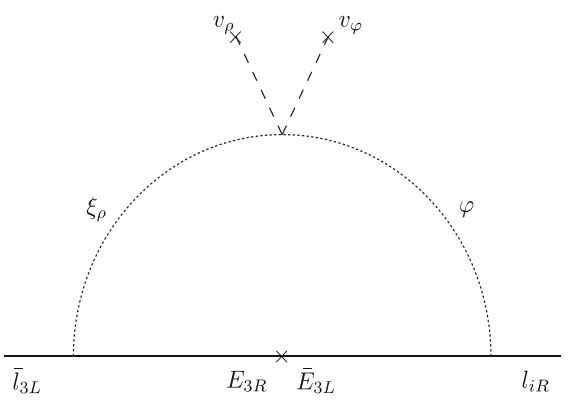

$$
\begin{aligned}
\tilde{M}_{l}= & \Delta_{l}+A_{l} \tilde{M}_{E}^{-1} B_{l} \\
& =\left(\begin{array}{ccc}
\varepsilon_{11}^{(l)} \frac{v_{\rho}}{\sqrt{2}} & \varepsilon_{12}^{(l)} \frac{v_{\rho}}{\sqrt{2}} & \varepsilon_{13}^{(l)} \frac{v_{\rho}}{\sqrt{2}} \\
\varepsilon_{21}^{(l)} \frac{v_{\rho}}{\sqrt{2}-x_{E} z_{1}^{(l)} \frac{v_{\xi} v_{\rho}}{\sqrt{2} m_{E}}} & \varepsilon_{22}^{(l)} \frac{v_{\rho}}{\sqrt{2}}-x_{E} z_{2}^{(l)} \frac{v_{\xi} v_{\rho}}{\sqrt{2} m_{E}} & \varepsilon_{23}^{(l)} \frac{v_{\rho}}{\sqrt{2}}-x_{E} z_{3}^{(l)} \frac{v_{\xi} v_{\rho}}{\sqrt{2} m_{E}} \\
\varepsilon_{31}^{(l)} \frac{v_{\rho}}{\sqrt{2}}+y_{E} x_{1}^{(l)} \frac{v_{\varphi} v_{\rho}}{\sqrt{2} m_{E_{3}}} & \varepsilon_{32}^{(l)} \frac{v_{\rho}}{\sqrt{2}}+y_{E} x_{2}^{(l)} \frac{v_{\varphi} v_{\rho}}{\sqrt{2} m_{E_{3}}} & \varepsilon_{33}^{(l)} \frac{v_{\rho}}{\sqrt{2}}+y_{E} x_{3}^{(l)} \frac{v_{\varphi} v_{\rho}}{\sqrt{2} m_{E_{3}}}
\end{array}\right) .
\end{aligned}
$$

In order to show that our model can naturally accommodate the experimental values of the charged lepton masses we use the extended benchmark scenario (26) assuming $m_{E}=m_{\tilde{T}}, m_{E_{3}}=m_{B_{3}}$. Then we have

$$
\begin{array}{rlrl}
v_{\xi} & =\lambda^{5} \frac{v m_{E}}{v_{\rho}}, & v_{\varphi}=\lambda^{3} \frac{v m_{E_{3}}}{v_{\rho}}, \\
\varepsilon_{i j}^{(l)}=b_{i j}^{(l)} \lambda^{9} \frac{v}{v_{\rho}}, & i, j=1,2,3 .
\end{array}
$$

Here the one-loop contributions $\varepsilon^{(l)}$ are estimated from their definitions in (31). Accordingly, the coefficients $b^{(l)}$ are constructed from the Yukawa and scalar quartic coupling. Thus, in the benchmark scenario (33) the SM charged lepton mass matrix reads:

$\tilde{M}_{l}=\left(\begin{array}{ccc}b_{11}^{(l)} \lambda^{9} & b_{12}^{(l)} \lambda^{9} & b_{13}^{(l)} \lambda^{9} \\ b_{21}^{(l)} \lambda^{9}+a_{21}^{(l)} \lambda^{5} & b_{22}^{(l)} \lambda^{9}+a_{22}^{(l)} \lambda^{5} & b_{23}^{(l)} \lambda^{9}+a_{23}^{(l)} \lambda^{5} \\ b_{31}^{(l)} \lambda^{9}+a_{31}^{(l)} \lambda^{3} & b_{32}^{(l)} \lambda^{9}+a_{32}^{(l)} \lambda^{3} & b_{33}^{(l)} \lambda^{9}+a_{33}^{(l)} \lambda^{3}\end{array}\right)$ $\frac{v}{\sqrt{2}}=\left(\begin{array}{ccc}c_{11}^{(l)} \lambda^{9} & c_{12}^{(l)} \lambda^{9} & c_{13}^{(l)} \lambda^{9} \\ c_{21}^{(l)} \lambda^{5} & c_{22}^{(l)} \lambda^{5} & c_{23}^{(l)} \lambda^{5} \\ c_{31}^{(l)} \lambda^{3} & c_{32}^{(l)} \lambda^{3} & c_{33}^{(l)} \lambda^{3}\end{array}\right) \frac{v}{\sqrt{2}}$

where

$$
\begin{array}{lll}
a_{21}^{(l)}=-x_{E} z_{1}^{(l)}, & a_{22}^{(l)}=-x_{E} z_{2}^{(l)}, & a_{23}^{(l)}=-x_{E} z_{3}^{(l),}, \\
a_{31}^{(l)}=y_{E} x_{1}^{(l)}, & a_{32}^{(l)}=y_{E} x_{2}^{(l)}, & a_{33}=y_{E} x_{3}^{(l)},
\end{array}
$$

$c_{21}^{(l)}=b_{21}^{(l)} \lambda^{4}+a_{21}^{(l)}, \quad c_{22}^{(l)}=b_{22}^{(l)} \lambda^{4}+a_{22}^{(l)}, \quad c_{23}^{(l)}=b_{23}^{(l)} \lambda^{4}+a_{23}^{(l)}$,

$c_{31}^{(l)}=b_{31}^{(l)} \lambda^{6}+a_{31}^{(l)}, \quad c_{32}^{(l)}=b_{32}^{(l)} \lambda^{6}+a_{32}^{(l)}, \quad c_{33}^{(l)}=b_{33}^{(l)} \lambda^{6}+a_{33}^{(l)}$,

$c_{1 i}^{(l)}=b_{1 i}^{(l)}, \quad i=1,2,3$.

The matrix in the second equality of Eq. (34) is shown for convenience in order to explicitly display the hierarchy of the matrix elements of $\tilde{M}_{l}$. To fit the measured values of the 
SM charged lepton masses [125], we solve the eigenvalue problem for the SM lepton mass matrix (34) and find the following solution:

$c_{i j}^{(l)}=\left(\begin{array}{ccc}-1.13637 & -1.03665 & -0.866907 \\ -0.658689 & -0.525883 & 1.08155 \\ 0.900883 & -0.32514 & -0.312796\end{array}\right)$

An important point is that all the elements of this matrix constructed from Yukawa couplings are $\sim O(1)$. This means that the observed hierarchical charged lepton mass spectrum can be naturally reproduced in our model without significant tuning of the coupling constants.

\section{Neutrino mass generation}

The neutrino Yukawa interactions give rise to the following neutrino mass terms:

$-\mathcal{L}_{\text {mass }}^{(v)}=\frac{1}{2}\left(\overline{v_{L}^{C}} \overline{\nu_{R}} \overline{N_{R}}\right) M_{\nu}\left(\begin{array}{c}v_{L} \\ v_{R}^{C} \\ N_{R}^{C}\end{array}\right)+H . c$,

where the neutrino mass matrix $M_{\nu}$ is

$M_{v}=\left(\begin{array}{ccc}M_{1} & 0_{3 \times 3} & 0_{3 \times 3} \\ 0_{3 \times 3} & M_{2} & M_{\chi} \\ 0_{3 \times 3} & M_{\chi}^{T} & \mu\end{array}\right)$,

with the submatrices $M_{1}$ and $M_{2}$ generated at one loop level, whereas the submatrices $M_{\chi}$ and $\mu$ appearing at tree level. They are given by:

$$
\begin{aligned}
M_{\chi} & =\left(\begin{array}{ccc}
x_{N} & 0 & 0 \\
0 & x_{N} & 0 \\
0 & 0 & y_{N}
\end{array}\right) \frac{v_{\chi}}{\sqrt{2}}, \\
\mu & =\left(\begin{array}{ccc}
h_{3 N} v_{\sigma_{3}}-h_{1 N} v_{\sigma_{1}} & h_{1 N} v_{\sigma_{2}} & h_{2 N} v_{\sigma_{2}} \\
h_{1 N} v_{\sigma_{2}} & h_{3 N} v_{\sigma_{3}}+h_{1 N} v_{\sigma_{1}} & -h_{2 N} v_{\sigma_{1}} \\
h_{2 N} v_{\sigma_{2}} & -h_{2 N} v_{\sigma_{1}} & h_{4 N} v_{\sigma_{3}}
\end{array}\right) .
\end{aligned}
$$

The light active neutrino mass matrix is generated by the loop diagrams shown in Fig. 5 and is given by:

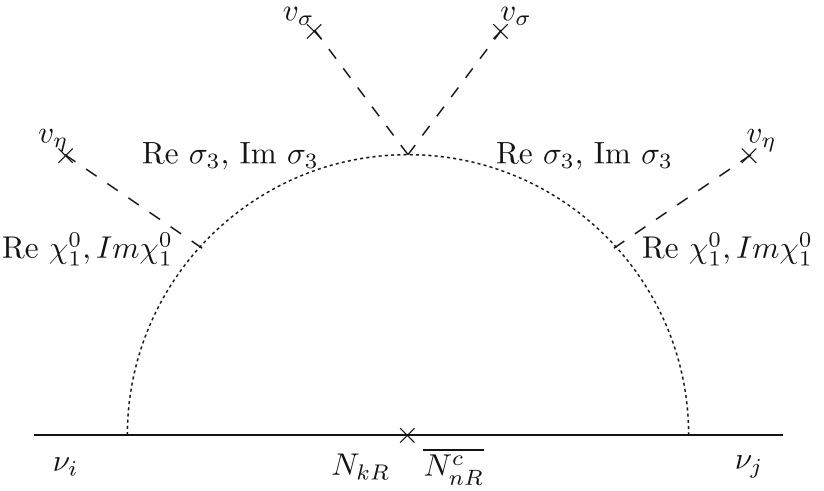

Fig. 5 One-loop Feynman diagram contributing to the entries of the light active neutrino mass matrix. Here $i, j, k, n=1,2,3$

with the loop function of the form [126]:

$$
\begin{aligned}
& F\left(m_{1}, m_{2}, m_{3}\right) \\
& =\frac{1}{16 \pi^{2}}\left[\frac{m_{2}^{2}}{m_{2}^{2}-m_{1}^{2}} \ln \left(\frac{m_{2}^{2}}{m_{1}^{2}}\right)-\frac{m_{3}^{2}}{m_{3}^{2}-m_{1}^{2}} \ln \left(\frac{m_{3}^{2}}{m_{1}^{2}}\right)\right] .
\end{aligned}
$$

In the limit where $\mu_{i j}^{2} \ll m_{\chi_{1 R}}^{2}, m_{\chi_{1 I}}^{2}$, the light active neutrino mass matrix becomes:

$$
\tilde{M}_{\nu} \simeq \frac{m_{\chi_{1 R}}^{2}-m_{\chi_{1 I}}^{2}}{8 \pi^{2}\left(m_{\chi_{1 R}}^{2}+m_{\chi_{1 I}}^{2}\right)}\left(\begin{array}{ccc}
x_{N}^{2} \mu_{22} & x_{N}^{2} \mu_{12} & x_{N} y_{N} \mu_{23} \\
x_{N}^{2} \mu_{12} & x_{N}^{2} \mu_{11} & x_{N} y_{N} \mu_{13} \\
x_{N} y_{N} \mu_{23} & x_{N} y_{N} \mu_{13} & y_{N}^{2} \mu_{33}
\end{array}\right) .
$$

All the elements of this mass matrix are free parameters and, therefore, our model does not predict specific values of neutrino masses and mixing. However, in our model, the small value of the overall neutrino mass scale is natural. As seen from Eq. (45), the smallness of this scale is attributed to a small splitting $\Delta m_{\chi}^{2}$ between the masses of the $\chi_{1 R}$ and $\chi_{1 I}$ scalar fields, which originates from the quartic term $\gamma\left(\chi^{\dagger} \eta \sigma_{3} \varphi+h . c\right)$, so that $\Delta m_{\chi}^{2} \sim \gamma v_{\varphi}^{2}$. (see Appendix B). Requiring smallness of the parameter $\kappa$, we must guarantee its stability with respect radiative corrections, i.e. its technical naturalness. Checking the model Lagrangian, we observe that in the limit $\gamma \rightarrow 0$ it acquires an extra symmetry, protecting this parameter from large radiative corrections. Here we do not need to specify this group completely and just give its minimal non-trivial subgroup. This is $Z_{3}$ with the field

$$
\tilde{M}_{\nu}=M_{1}=\left(\begin{array}{ccc}
x_{N}^{2} F\left(\mu_{22}, m_{\chi_{1 R}}, m_{\chi_{1 I}}\right) \mu_{22} & -x_{N}^{2} F\left(\mu_{12}, m_{\chi_{1 R}}, m_{\chi_{1 I}}\right) \mu_{12} & x_{N} y_{N} F\left(\mu_{23}, m_{\chi_{1 R}}, m_{\chi_{1 I}}\right) \mu_{23} \\
-x_{N}^{2} F\left(\mu_{12}, m_{\chi_{1 R}}, m_{\chi_{1 I}}\right) \mu_{12} & x_{N}^{2} F\left(\mu_{11}, m_{\chi_{1 R}}, m_{\chi_{1 I}}\right) \mu_{11} & -x_{N} y_{N} F\left(\mu_{13}, m_{\chi_{1 R}}, m_{\chi_{1 I}}\right) \mu_{13} \\
x_{N} y_{N} F\left(\mu_{23}, m_{\chi_{1 R}}, m_{\chi_{1 I}}\right) \mu_{23}-x_{N} y_{N} F\left(\mu_{13}, m_{\chi_{1 R}}, m_{\chi_{1 I}}\right) \mu_{13} & y_{N}^{2} F\left(\mu_{33}, m_{\chi_{1 R}}, m_{\chi_{1 I}}\right) \mu_{33}
\end{array}\right),
$$


assignment, where all leptonic fields as well as the scalar fields $\sigma$ and $\sigma_{3}$ have a charge equal to $\omega=e^{\frac{2 \pi i}{3}}$, whereas the remaining fields are neutral under this symmetry. This symmetry is broken by the coupling $\gamma$. Therefore, in our model small masses of the light neutrinos are technically natural, being protected by this accidental symmetry. As a result, the components $\chi_{1,2}$ of the scalar $S U(3)_{L}$-triplet can be sufficiently light to provide a non-trivial phenomenology.

\section{Conclusions}

We have constructed a renormalizable theory based on the $S U(3)_{C} \times S U(3)_{L} \times U(1)_{X}$ gauge symmetry, supplemented with the spontaneously broken $U(1)_{L_{g}}$ global lepton number symmetry and the $S_{3} \times Z_{2}$ discrete group, consistent with the low energy SM fermion flavor data. In our model, the particle spectrum of the 3-3-1 model with right handed Majorana neutrinos is enlarged by the inclusion of gauge singlet scalars and charged exotic vector like fermions, which are crucial for the implementation of the tree level Universal seesaw mechanism that produces the masses for the bottom, strange and charm quarks as well as the tau and muon lepton masses. The top and exotic quarks obtain their tree level masses from renormalizable Yukawa interactions, whereas the first generation SM charged fermion masses are generated from a one loop level radiative seesaw mechanism. The masses for the light active neutrinos arise from a radiative seesaw mechanism at one loop level. The natural smallness of the overall neutrino mass scale is guarantied by an accidental softly broken symmetry. Our model successfully explains the hierarchy of the fermion masses and mixings as well as accommodates the current experimental deviations of the electron and muon anomalous magnetic moments from their SM values.

Acknowledgements This research has received funding from Fondecyt (Chile), Grants no. 1170803, no. 1190845, ANID PIA/APOYO AFB180002, the Vietnam National Foundation for Science and Technology Development (NAFOSTED) under grant number 103.012017.341. A.E.C.H is very grateful to the Institute of Physics, Vietnam Academy of Science and Technology for the warm hospitality and for financing his visit.

Data Availability Statement This manuscript has no associated data or the data will not be deposited. [Authors' comment: The only experimental data used in our work are the ones reported in Eqs. (10), (11) and Table 3 as well as the experimental values of the charged lepton masses in Ref. [125] that we use to compare the predictions of our model in SM fermion masses and mixings, and electron and muon anomalous magnetic dipole moments with the experimental data.]

Open Access This article is licensed under a Creative Commons Attribution 4.0 International License, which permits use, sharing, adaptation, distribution and reproduction in any medium or format, as long as you give appropriate credit to the original author(s) and the source, provide a link to the Creative Commons licence, and indicate if changes were made. The images or other third party material in this article are included in the article's Creative Commons licence, unless indicated otherwise in a credit line to the material. If material is not included in the article's Creative Commons licence and your intended use is not permitted by statutory regulation or exceeds the permitted use, you will need to obtain permission directly from the copyright holder. To view a copy of this licence, visit http://creativecomm ons.org/licenses/by/4.0/.

Funded by $\mathrm{SCOAP}^{3}$.

\section{Appendix A: The $S_{3}$ discrete group}

The $S_{3}$ discrete group contains 3 irreducible representations: $\mathbf{1}, \mathbf{1}^{\prime}$ and $\mathbf{2}$. Considering $\left(x_{1}, x_{2}\right)^{T}$ and $\left(y_{1}, y_{2}\right)^{T}$ as the basis vectors for two $S_{3}$ doublets and ( $\left.y\right)$ is an $S_{3}$ non trivial singlet, the multiplication rules of the $S_{3}$ group for the case of real representations take the form [127]:

$$
\begin{aligned}
&\left(\begin{array}{l}
x_{1} \\
x_{2}
\end{array}\right)_{\mathbf{2}} \otimes\left(\begin{array}{l}
y_{1} \\
y_{2}
\end{array}\right)_{\mathbf{2}}=\left(x_{1} y_{1}+x_{2} y_{2}\right)_{\mathbf{1}}+\left(x_{1} y_{2}-x_{2} y_{1}\right)_{\mathbf{1}^{\prime}} \\
&+\left(\begin{array}{c}
x_{2} y_{2}-x_{1} y_{1} \\
x_{1} y_{2}+x_{2} y_{1}
\end{array}\right)_{\mathbf{2}} \\
&\left(\begin{array}{l}
x_{1} \\
x_{2}
\end{array}\right)_{\mathbf{2}} \otimes(y)_{\mathbf{1}^{\prime}}=\left(\begin{array}{c}
-x_{2} y^{\prime} \\
x_{1} y^{\prime}
\end{array}\right)_{\mathbf{2}} \\
&(x)_{\mathbf{1}^{\prime}} \otimes(y)_{\mathbf{1}^{\prime}}=\left(x^{\prime} y\right)_{\mathbf{1}} .
\end{aligned}
$$

\section{Appendix B: The scalar potential}

The renormalizable scalar potential of our model takes the form:

$$
\begin{aligned}
V= & -\mu_{\chi}^{2}\left(\chi^{\dagger} \chi\right)-\mu_{\eta}^{2}\left(\eta^{\dagger} \eta\right)-\mu_{\rho}^{2}\left(\rho^{\dagger} \rho\right)-\mu_{\xi}^{2}(\xi \xi)_{\mathbf{1}} \\
& -\mu_{\varphi}^{2} \varphi^{2}-\mu_{\sigma}^{2}\left(\sigma^{\dagger} \sigma\right)_{\mathbf{1}}-\mu_{\sigma_{3}}^{2}\left(\sigma_{3}^{\dagger} \sigma_{3}\right) \\
& +\left(\kappa \varphi \eta_{i} \chi_{j} \rho_{k} \varepsilon^{i j k}+H . c .\right)+\gamma\left(\chi^{\dagger} \eta \sigma_{3} \varphi+h . c\right) \\
& +\lambda_{1}\left(\chi^{\dagger} \chi\right)\left(\chi^{\dagger} \chi\right)+\lambda_{2}\left(\rho^{\dagger} \rho\right)\left(\rho^{\dagger} \rho\right)+\lambda_{3}\left(\eta^{\dagger} \eta\right)\left(\eta^{\dagger} \eta\right) \\
& +\lambda_{4}\left(\chi^{\dagger} \chi\right)\left(\rho^{\dagger} \rho\right)+\lambda_{5}\left(\chi^{\dagger} \chi\right)\left(\eta^{\dagger} \eta\right)+\lambda_{6}\left(\rho^{\dagger} \rho\right)\left(\eta^{\dagger} \eta\right) \\
& +\lambda_{7}\left(\chi^{\dagger} \eta\right)\left(\eta^{\dagger} \chi\right)+\lambda_{8}\left(\chi^{\dagger} \rho\right)\left(\rho^{\dagger} \chi\right)+\lambda_{9}\left(\rho^{\dagger} \eta\right)\left(\eta^{\dagger} \rho\right) \\
& +\lambda_{10}\left[(\xi \xi)_{1}\right]^{2}+\lambda_{11}\left[(\xi \xi)_{\mathbf{1}^{\prime}}\right]^{2}+\lambda_{12}\left[(\xi \xi)_{\mathbf{2}}(\xi \xi)_{\mathbf{2}}\right]_{\mathbf{1}} \\
& +\lambda_{13} \varphi^{4}+\lambda_{14}\left[\left(\sigma^{\dagger} \sigma\right)_{\mathbf{1}}\right]^{2}+\lambda_{15}\left[\left(\sigma^{\dagger} \sigma\right)_{\mathbf{1}^{\prime}}\right]^{2} \\
& +\lambda_{16}\left[\left(\sigma^{\dagger} \sigma\right)_{\mathbf{2}}\right]^{2} \\
& +\lambda_{17}\left(\sigma_{3}^{\dagger} \sigma_{3}\right)^{2}+\lambda_{18}(\xi \xi)_{\mathbf{1}} \varphi^{2}+\lambda_{19}\left[(\xi \xi)_{\mathbf{1}}\right]\left(\sigma^{\dagger} \sigma\right)_{\mathbf{1}} \\
& +\lambda_{20}\left[(\xi \xi)_{\mathbf{1}^{\prime}}\right]\left(\sigma^{\dagger} \sigma\right)_{\mathbf{1}^{\prime}}+\lambda_{21}\left[(\xi \xi)_{2}\right]\left(\sigma^{\dagger} \sigma\right)_{\mathbf{2}} \\
& +\lambda_{22}(\xi \xi)_{\mathbf{1}}\left(\sigma_{3}^{\dagger} \sigma_{3}\right)+\lambda_{23} \varphi^{2}\left(\sigma^{\dagger} \sigma\right)_{\mathbf{1}}+\lambda_{24}\left(\sigma^{\dagger} \sigma\right)_{\mathbf{1}}\left(\sigma_{3}^{\dagger} \sigma_{3}\right) \\
& +\lambda_{25}\left[(\sigma \sigma)_{\mathbf{1}}\left(\sigma_{3}^{\dagger} \sigma_{3}^{\dagger}\right)+h . c\right]+\lambda_{26} \varphi^{2}\left(\chi^{\dagger} \chi\right) \\
& +\lambda_{27}(\xi \xi)_{\mathbf{1}}\left(\chi^{\dagger} \chi\right)+\lambda_{28}\left(\sigma^{\dagger} \sigma\right)_{\mathbf{1}}\left(\chi^{\dagger} \chi\right) \\
& +\lambda_{29}\left(\sigma_{3}^{\dagger} \sigma_{3}\right)\left(\chi^{\dagger} \chi\right)+\lambda_{30}(\xi \xi)_{\mathbf{1}}\left(\eta^{\dagger} \eta\right)+\lambda_{31} \varphi^{2}\left(\eta^{\dagger} \eta\right)
\end{aligned}
$$




$$
\begin{aligned}
& +\lambda_{32}\left(\sigma^{\dagger} \sigma\right)_{1}\left(\eta^{\dagger} \eta\right)+\lambda_{33}\left(\sigma_{3}^{\dagger} \sigma_{3}\right)\left(\eta^{\dagger} \eta\right) \\
& +\lambda_{34}(\xi \xi)_{1}\left(\rho^{\dagger} \rho\right)+\lambda_{35} \varphi^{2}\left(\rho^{\dagger} \rho\right)+\lambda_{36}\left(\sigma^{\dagger} \sigma\right)_{1}\left(\rho^{\dagger} \rho\right) \\
& +\lambda_{37}\left(\sigma_{3}^{\dagger} \sigma_{3}\right)\left(\rho^{\dagger} \rho\right)+\lambda_{38}\left\{\left[(\xi \xi)_{2} \xi\right]_{\mathbf{1}^{\prime}} \varphi+h . c\right\} \\
& +\lambda_{39}\left[(\xi \xi)_{2}\left(\sigma^{\dagger} \sigma_{3}\right)+h . c\right] \\
& +\lambda_{40}\left[\left(\sigma^{\dagger} \xi\right)_{\mathbf{1}^{\prime}} \varphi \sigma_{3}+\text { h.c }\right]
\end{aligned}
$$

where $\lambda_{i}(i=1,2, \ldots, 40)$ are dimensionless parameters, whereas $\mu_{\chi}, \mu_{\eta}, \mu_{\xi}, \mu_{\varphi}, \mu_{\sigma}, \mu_{\sigma_{3}}, f$ and $A$ are dimensionful parameters. Here $\chi, \rho$ and $\eta$, the $S U(3)_{L}$ scalar triplets and the remaining fields are $S U(3)_{L}$ scalar singlets. The scalar fields $\sigma$ and $\sigma_{3}$ are complex, wheras $\xi$ and $\varphi$ are real.

\section{Appendix C: The scalar potential and scalar mass matrix entries for the $\mathrm{g}-2$ contribution}

The scalar potential that contributes to $g-2$ anomalies is given by:

$$
\begin{aligned}
V^{\mathrm{CP}}= & -\mu_{\rho}^{2}\left(\rho^{\dagger} \rho\right)-\mu_{\xi}^{2}(\xi \xi)_{1}-\mu_{\varphi}^{2} \varphi^{2} \\
& +\left(\kappa \varphi \eta_{i} \chi_{j} \rho_{k} \varepsilon^{i j k}+H . c .\right) \\
& +\lambda_{2}\left(\rho^{\dagger} \rho\right)\left(\rho^{\dagger} \rho\right)+\lambda_{10}\left[(\xi \xi)_{1}\right]^{2}+\lambda_{11}\left[(\xi \xi)_{1^{\prime}}\right]^{2} \\
& +\lambda_{12}\left[(\xi \xi)_{2}(\xi \xi)_{2}\right]_{1}+\lambda_{13} \varphi^{4}+\lambda_{18}(\xi \xi)_{1} \varphi^{2} \\
& +\lambda_{34}(\xi \xi)_{1}\left(\rho^{\dagger} \rho\right)+\lambda_{35} \varphi^{2}\left(\rho^{\dagger} \rho\right) \\
& +\lambda_{38}\left\{\left[(\xi \xi)_{2} \xi\right]_{\mathbf{1}^{\prime}} \varphi+h . c\right\}
\end{aligned}
$$

with the complex quartic coupling $\kappa=\kappa_{r}+i \kappa_{i}$ introducing $\mathrm{CP}$-violation in the scalar potential. We need it in order to $\operatorname{mix} \varphi$ with $\zeta_{\rho}$. The scalar potential minimization equations allow us to express the $\mu_{\rho}, \mu_{\xi}$ and $\mu_{\varphi}$ parameters as follows:

$$
\begin{aligned}
\mu_{\rho}^{2} & =\frac{1}{2}\left(\frac{\kappa_{r} v_{\eta} v_{\varphi} v_{\chi}}{v_{\rho}}+\lambda_{34} v_{\xi}^{2}+2 \lambda_{2} v_{\rho}^{2}+\lambda_{35} v_{\varphi}^{2}\right) \\
\mu_{\xi}^{2} & =\frac{1}{2}\left(2\left(\lambda_{10}+\lambda_{12}\right) v_{\xi}^{2}+\lambda_{34} v_{\rho}^{2}+\lambda_{18} v_{\varphi}^{2}\right) \\
\mu_{\varphi}^{2} & =\frac{1}{2}\left(\frac{\kappa_{r} v_{\eta} v_{\rho} v_{\chi}}{v_{\varphi}}+\lambda_{18} v_{\xi}^{2}+\lambda_{35} v_{\rho}^{2}+2 \lambda_{13} v_{\varphi}^{2}\right)
\end{aligned}
$$

The potential (C1) generates the entries of the scalar mass squared matrix $\mathbf{M}^{2}$. In the basis $\xi_{\rho}, \xi_{1 R}, \xi_{2 R}, \varphi, \zeta_{\rho}$ these entries are given by:

$$
\begin{aligned}
& m_{11}^{2}=2 \lambda_{2} v_{\rho}^{2}-\frac{\kappa_{r} v_{\eta} v_{\varphi} v_{\chi}}{2 v_{\rho}} \\
& m_{12}^{2}=m_{21}^{2}=\lambda_{34} v_{\xi} v_{\rho} \\
& m_{14}^{2}=m_{41}^{2}=\frac{1}{2} \kappa_{r} v_{\eta} v_{\chi}+\lambda_{35} v_{\rho} v_{\varphi} \\
& m_{22}^{2}=2\left(\lambda_{10}+\lambda_{12}\right) v_{\xi}^{2} \\
& m_{23}^{2}=m_{32}^{2}=-2 \sqrt{2} \lambda_{38} v_{\xi} v_{\varphi}
\end{aligned}
$$

$m_{24}^{2}=m_{42}^{2}=\lambda_{18} v_{\xi} v_{\varphi}$

$m_{33}^{2}=2 \lambda_{10} v_{\xi}^{2}-3 \lambda_{12} v_{\xi}^{2}-2 \lambda_{38} v_{\varphi} v_{\xi}$

$m_{34}^{2}=m_{43}^{2}=-\sqrt{2} \lambda_{38} v_{\xi}^{2}$

$m_{44}^{2}=2 \lambda_{13} v_{\varphi}^{2}-\frac{\kappa_{r} v_{\eta} v_{\rho} v_{\chi}}{2 v_{\varphi}}$

$m_{45}^{2}=m_{54}^{2}=-\frac{1}{2} \kappa_{i} v_{\eta} v_{\chi}$

$m_{55}^{2}=-\frac{\kappa_{r} v_{\eta} v_{\varphi} v_{\chi}}{2 v_{\rho}}$.

From these expressions one can see that by the appropriate choice of the signs of the quartic couplings it easy to guarantee positive definiteness of the mass squared matrix (12) and, therefore, that the extremum conditions (C2)-(C4) correspond to the minimum of the potential $(\mathrm{C} 1)$.

\section{References}

1. H. Georgi, A. Pais, Generalization of gim: horizontal and vertical flavor mixing. Phys. Rev. D 19, 2746 (1979). https://doi.org/10. 1103/PhysRevD.19.2746

2. J.W.F. Valle, M. Singer, Lepton number violation with quasi dirac neutrinos. Phys. Rev. D 28, 540 (1983). https://doi.org/10.1103/ PhysRevD.28.540

3. F. Pisano, V. Pleitez, An SU(3) x U(1) model for electroweak interactions. Phys. Rev. D 46, 410-417 (1992). https://doi.org/ 10.1103/PhysRevD.46.410. arXiv:hep-ph/9206242

4. R. Foot, O.F. Hernandez, F. Pisano, V. Pleitez, Lepton masses in an SU(3)-L x U(1)-N gauge model. Phys. Rev. D 47, 4158-4161 (1993). https://doi.org/10.1103/PhysRevD.47.4158. arXiv:hep-ph/9207264

5. P.H. Frampton, Chiral dilepton model and the flavor question. Phys. Rev. Lett. 69, 2889-2891 (1992). https://doi.org/10.1103/ PhysRevLett.69.2889

6. H.N. Long, SU(3)-L x U(1)-N model for right-handed neutrino neutral currents. Phys. Rev. D 54, 4691-4693 (1996). https://doi. org/10.1103/PhysRevD.54.4691. arXiv:hep-ph/9607439

7. H.N. Long, The 331 model with right handed neutrinos. Phys. Rev. D 53, 437-445 (1996). https://doi.org/10.1103/PhysRevD. 53.437. arXiv:hep-ph/9504274

8. R. Foot, H.N. Long, T.A. Tran, $S U(3)_{L} \otimes U(1)_{N}$ and $S U(4)_{L} \otimes$ $U(1)_{N}$ gauge models with right-handed neutrinos. Phys. Rev. D 50(1), R34-R38 (1994). https://doi.org/10.1103/PhysRevD.50. R34. arXiv:hep-ph/9402243

9. A.E. Carcamo Hernandez, R. Martinez, F. Ochoa, Z and Z' decays with and without FCNC in 331 models. Phys. Rev. D 73, 035007 (2006). https://doi.org/10.1103/PhysRevD.73. 035007. arXiv:hep-ph/0510421

10. P.V. Dong, H.N. Long, D.V. Soa, V.V. Vien, The 3-3-1 model with $S_{4}$ flavor symmetry. Eur. Phys. J. C 71, 1544 (2011). https://doi. org/10.1140/epjc/s10052-011-1544-2. arXiv:1009.2328 [hep$\mathrm{ph}]$

11. P.V. Dong, L.T. Hue, H.N. Long, D.V. Soa, The 3-3-1 model with $\mathrm{A}_{4}$ flavor symmetry. Phys. Rev. D 81, 053004 (2010). https://doi. org/10.1103/PhysRevD.81.053004. arXiv:1001.4625 [hep-ph]

12. P.V. Dong, H.N. Long, C.H. Nam, V.V. Vien, The $S_{3}$ flavor symmetry in 3-3-1 models. Phys. Rev. D 85, 053001 (2012). https://doi. org/10.1103/PhysRevD.85.053001. arXiv:1111.6360 [hep-ph] 
13. R.H. Benavides, W.A. Ponce, Y. Giraldo, $S U(3)_{c} \otimes S U(3)_{L} \otimes$ $U(1)_{X}$ models with four families. Phys. Rev. D 82, 013004 (2010). https://doi.org/10.1103/PhysRevD.82.013004. arXiv:1006.3248 [hep-ph]

14. P.V. Dong, H.N. Long, H.T. Hung, Question of Peccei-Quinn symmetry and quark masses in the economical 3-3-1 model. Phys. Rev. D 86, 033002 (2012). https://doi.org/10.1103/PhysRevD.86. 033002. arXiv:1205.5648 [hep-ph]

15. D.T. Huong, L.T. Hue, M.C. Rodriguez, H.N. Long, Supersymmetric reduced minimal 3-3-1 model. Nucl. Phys. B 870, 293-322 (2013). https://doi.org/10.1016/j.nuclphysb.2013. 01.016. arXiv:1210.6776 [hep-ph]

16. P.T. Giang, L.T. Hue, D.T. Huong, H.N. Long, Leptonflavor violating decays of neutral Higgs to muon and tauon in supersymmetric economical 3-3-1 model. Nucl. Phys. B 864, 85-112 (2012). https://doi.org/10.1016/j.nuclphysb.2012. 06.008. arXiv:1204.2902 [hep-ph]

17. D.T. Binh, L.T. Hue, D.T. Huong, H.N. Long, Higgs revised in supersymmetric economical 3-3-1 model with $B / \mu$-type terms. Eur. Phys. J. C 74(5), 2851 (2014). https://doi.org/10.1140/epjc/ s10052-014-2851-1. arXiv:1308.3085 [hep-ph]

18. A.E. Carcamo Hernandez, R. Martinez, F. Ochoa, Radiative seesaw-type mechanism of quark masses in $S U(3)_{C} \otimes S U(3)_{L} \otimes$ $U(1)_{X}$. Phys. Rev. D 87(7), 075009 (2013). https://doi.org/10. 1103/PhysRevD.87.075009. arXiv:1302.1757 [hep-ph]

19. A.E. Cárcamo Hernández, R. Martinez, F. Ochoa, Fermion masses and mixings in the 3-3-1 model with right-handed neutrinos based on the $S_{3}$ flavor symmetry. Eur. Phys. J. C 76(11), 634 (2016). https://doi.org/10.1140/epjc/s10052-016-4480-3. arXiv: 1309.6567 [hep-ph]

20. A.E. Cárcamo Hernández, R. Martinez, J. Nisperuza, $S_{3}$ discrete group as a source of the quark mass and mixing pattern in 331 models. Eur. Phys. J. C 75(2), 72 (2015). https://doi.org/10.1140/ epjc/s10052-015-3278-z. arXiv:1401.0937 [hep-ph]

21. A.E. Cárcamo Hernández, E. Catañ oMur, R. Martinez, Lepton masses and mixing in $S U(3)_{C} \otimes S U(3)_{L} \otimes U(1)_{X}$ models with a $S_{3}$ flavor symmetry. Phys. Rev. D 90(7), 073001 (2014). https://doi.org/10.1103/PhysRevD.90.073001. arXiv:1407.5217 [hep-ph]

22. C. Kelso, H.N. Long, R. Martinez, F.S. Queiroz, Connection of $g-2 \mu$, electroweak, dark matter, and collider constraints on 331 models. Phys. Rev. D 90(11), 113011 (2014). https://doi.org/10. 1103/PhysRevD.90.113011. arXiv:1408.6203 [hep-ph]

23. V.V. Vien, H.N. Long, The $T_{7}$ flavor symmetry in 3-3-1 model with neutral leptons. JHEP 04, 133 (2014). https://doi.org/10.1007/ JHEP04(2014)133. arXiv:1402.1256 [hep-ph]

24. V.Q. Phong, H.N. Long, V.T. Van, L.H. Minh, Electroweak phase transition in the economical 3-3-1 model. Eur. Phys. J. C 75(7), 342 (2015). https://doi.org/10.1140/epjc/s10052-015-3550-2. arXiv: 1409.0750 [hep-ph]

25. V.Q. Phong, H.N. Long, V.T. Van, N.C. Thanh, Electroweak sphalerons in the reduced minimal 3-3-1 model. Phys. Rev. D 90(8), 085019 (2014). https://doi.org/10.1103/PhysRevD.90. 085019. arXiv:1408.5657 [hep-ph]

26. S.M. Boucenna, S. Morisi, J.W.F. Valle, Radiative neutrino mass in 3-3-1 scheme. Phys. Rev. D 90(1), 013005 (2014). https://doi. org/10.1103/PhysRevD.90.013005. arXiv:1405.2332 [hep-ph]

27. G. De Conto, A.C.B. Machado, V. Pleitez, Minimal 3-3-1 model with a spectator sextet. Phys. Rev. D 92(7), 075031 (2015). https:// doi.org/10.1103/PhysRevD.92.075031. arXiv:1505.01343 [hep$\mathrm{ph}]$

28. S.M. Boucenna, J.W.F. Valle, A. Vicente, Predicting charged lepton flavor violation from 3-3-1 gauge symmetry. Phys. Rev. D 92(5), 053001 (2015). https://doi.org/10.1103/PhysRevD.92. 053001. arXiv:1502.07546 [hep-ph]
29. S.M. Boucenna, S. Morisi, A. Vicente, The LHC diphoton resonance from gauge symmetry. Phys. Rev. D 93(11), 115008 (2016). https://doi.org/10.1103/PhysRevD.93.115008. arXiv: 1512.06878 [hep-ph]

30. R.H. Benavides, L.N. Epele, H. Fanchiotti, C.G. Canal, W.A. Ponce, Lepton number violation and neutrino masses in 3-3-1 models. Adv. High Energy Phys. 2015, 813129 (2015). https:// doi.org/10.1155/2015/813129. arXiv:1503.01686 [hep-ph]

31. A.E. Cárcamo Hernández, R. Martinez, A predictive 3-31 model with $A_{4}$ flavor symmetry. Nucl. Phys. B 905, 337-358 (2016). https://doi.org/10.1016/j.nuclphysb.2016.02. 025. arXiv:1501.05937 [hep-ph]

32. A.E. Cárcamo Hernández, R. Martinez, Fermion mass and mixing pattern in a minimal T7 flavor 331 model. J. Phys. G 43(4), 045003 (2016). https://doi.org/10.1088/0954-3899/43/4/ 045003. arXiv:1501.07261 [hep-ph]

33. L.T. Hue, H.N. Long, T.T. Thuc, T. Phong Nguyen, Lepton flavor violating decays of Standard-Model-like Higgs in 3-3-1 model with neutral lepton. Nucl. Phys. B 907, 37-76 (2016). https://doi. org/10.1016/j.nuclphysb.2016.03.034. arXiv:1512.03266 [hep$\mathrm{ph}]$

34. A.E.C. Hernández, I. Nišandžić, LHC diphoton resonance at 750 $\mathrm{GeV}$ as an indication of $S U(3)_{L} \times U(1)_{X}$ electroweak symmetry. Eur. Phys. J. C 76(7), 380 (2016). https://doi.org/10.1140/epjc/ s10052-016-4230-6. arXiv:1512.07165 [hep-ph]

35. R.M. Fonseca, M. Hirsch, A flipped 331 model. JHEP 08, 003 (2016). https://doi.org/10.1007/JHEP08(2016)003. arXiv:1606.01109 [hep-ph]

36. V.V. Vien, A.E. Cárcamo Hernández, H.N. Long, The $\Delta(27)$ flavor 3-3-1 model with neutral leptons. Nucl. Phys. B 913, 792-814 (2016). https://doi.org/10.1016/j.nuclphysb.2016.10. 010. arXiv:1601.03300 [hep-ph]

37. A.E. Cárcamo Hernández, H.N. Long, V.V. Vien, A 3-3-1 model with right-handed neutrinos based on the $\Delta(27)$ family symmetry. Eur. Phys. J. C 76(5), 242 (2016). https://doi.org/10.1140/epjc/ s10052-016-4074-0. arXiv:1601.05062 [hep-ph]

38. R.M. Fonseca, M. Hirsch, Lepton number violation in 331 models. Phys. Rev. D 94(11), 115003 (2016). https://doi.org/10.1103/ PhysRevD.94.115003. arXiv:1607.06328 [hep-ph]

39. F.F. Deppisch, C. Hati, S. Patra, U. Sarkar, J.W.F. Valle, 331 models and grand unification: from minimal $\mathrm{SU}(5)$ to minimal SU(6). Phys. Lett. B 762, 432-440 (2016). https://doi.org/10. 1016/j.physletb.2016.10.002. arXiv:1608.05334 [hep-ph]

40. M. Reig, J.W.F. Valle, C.A. Vaquera-Araujo, Realistic SU(3) $\mathrm{c}_{\mathrm{c}} \otimes$ $\mathrm{SU}(3)_{\mathrm{L}} \otimes \mathrm{U}(1)_{\mathrm{X}}$ model with a type II Dirac neutrino seesaw mechanism. Phys. Rev. D 94(3), 033012 (2016). https://doi.org/ 10.1103/PhysRevD.94.033012. arXiv:1606.08499 [hep-ph]

41. A.E. Cárcamo Hernández, S. Kovalenko, H.N. Long, I. Schmidt, A variant of 3-3-1 model for the generation of the SM fermion mass and mixing pattern. JHEP 07, 144 (2018). https://doi.org/ 10.1007/JHEP07(2018)144. arXiv:1705.09169 [hep-ph]

42. A.E. Cárcamo Hernández, H.N. Long, A highly predictive $A_{4}$ flavour 3-3-1 model with radiative inverse seesaw mechanism. J. Phys. G 45(4), 045001 (2018). https://doi.org/10.1088/ 1361-6471/aaace7. arXiv:1705.05246 [hep-ph]

43. C. Hati, S. Patra, M. Reig, J.W.F. Valle, C.A. Vaquera-Araujo, Towards gauge coupling unification in left-right symmetric $\mathrm{SU}(3)_{\mathrm{c}} \times \mathrm{SU}(3)_{\mathrm{L}} \times \mathrm{SU}(3)_{\mathrm{R}} \times \mathrm{U}(1)_{\mathrm{X}}$ theories. Phys. Rev. D 96(1), 015004 (2017). https://doi.org/10.1103/PhysRevD.96. 015004. arXiv:1703.09647 [hep-ph]

44. E.R. Barreto, A.G. Dias, J. Leite, C.C. Nishi, R.L.N. Oliveira, W.C. Vieira, Hierarchical fermions and detectable $Z^{\prime}$ from effective two-Higgs-triplet 3-3-1 model. Phys. Rev. D 97(5), 055047 (2018). https://doi.org/10.1103/PhysRevD.97.055047. arXiv:1709.09946 [hep-ph] 
45. A.E. Cárcamo Hernández, H.N. Long, V.V. Vien, The first $\Delta$ (27) flavor 3-3-1 model with low scale seesaw mechanism. Eur. Phys. J. C 78(10), 804 (2018). https://doi.org/10.1140/epjc/ s10052-018-6284-0. arXiv:1803.01636 [hep-ph]

46. V.V. Vien, H.N. Long, A.E. Cárcamo Hernández, Lepton masses and mixings in a $T^{\prime}$ flavoured 3-3-1 model with type I and II seesaw mechanisms. Mod. Phys. Lett. A 34(01), 1950005 (2019). https://doi.org/10.1142/S0217732319500056. arXiv:1812.07263 [hep-ph]

47. A.G. Dias, J. Leite, D.D. Lopes, C.C. Nishi, Fermion mass hierarchy and double seesaw mechanism in a 3-3-1 model with an axion. Phys. Rev. D 98(11), 115017 (2018). https://doi.org/10. 1103/PhysRevD.98.115017. arXiv:1810.01893 [hep-ph]

48. M.M. Ferreira, T.B. de Melo, S. Kovalenko, P.R.D. Pinheiro, F.S. Queiroz, Lepton flavor violation and collider searches in a type I + II seesaw model. Eur. Phys. J. C 79(11), 955 (2019). https://doi. org/10.1140/epjc/s10052-019-7422-z. arXiv:1903.07634 [hep$\mathrm{ph}]$

49. A.E. Cárcamo Hernández, Y. Hidalgo Velásquez, N.A. PérezJulve, A 3-3-1 model with low scale seesaw mechanisms. Eur. Phys. J. C 79(10), 828 (2019). https://doi.org/10.1140/epjc/ s10052-019-7325-z. arXiv:1905.02323 [hep-ph]

50. A.E. Cárcamo Hernández, N.A. Pérez-Julve, Y. Hidalgo Velásquez, Fermion masses and mixings and some phenomenological aspects of a 3-3-1 model with linear seesaw mechanism. Phys. Rev. D 100(9), 095025 (2019). https://doi.org/10.1103/ PhysRevD.100.095025. arXiv:1907.13083 [hep-ph]

51. A.E. Cárcamo Hernández, D.T. Huong, H.N. Long, Minimal model for the fermion flavor structure, mass hierarchy, dark matter, leptogenesis, and the electron and muon anomalous magnetic moments. Phys. Rev. D 102(5), 055002 (2020). https://doi.org/ 10.1103/PhysRevD.102.055002. arXiv:1910.12877 [hep-ph]

52. A.E. Cárcamo Hernández, L.T. Hue, S. Kovalenko, H.N. Long, An extended 3-3-1 model with two scalar triplets and linear seesaw mechanism. arXiv:2001.01748 [hep-ph]

53. A. Crivellin, M. Hoferichter, P. Schmidt-Wellenburg, Combined explanations of $(g-2)_{\mu, e}$ and implications for a large muon EDM. Phys. Rev. D 98(11), 113002 (2018). https://doi.org/10. 1103/PhysRevD.98.113002. arXiv:1807.11484 [hep-ph]

54. M. Endo, W. Yin, Explaining electron and muon $g-2$ anomaly in SUSY without lepton-flavor mixings. JHEP 08, 122 (2019). https://doi.org/10.1007/JHEP08(2019)122. arXiv:1906.08768 [hep-ph]

55. G.F. Giudice, P. Paradisi, M. Passera, Testing new physics with the electron g-2. JHEP 11, 113 (2012). https://doi.org/10.1007/ JHEP11(2012)113. arXiv:1208.6583 [hep-ph]

56. A. Falkowski, S.F. King, E. Perdomo, M. Pierre, Flavourful $Z^{\prime}$ portal for vector-like neutrino Dark Matter and $R_{K^{(*)}}$. JHEP 08, 061 (2018). https://doi.org/10.1007/JHEP08(2018)061. arXiv:1803.04430 [hep-ph]

57. B. Allanach, F.S. Queiroz, A. Strumia, S. Sun, $Z^{\prime}$ models for the LHCb and $g-2$ muon anomalies. Phys. Rev. D 93(5), 055045 (2016). https://doi.org/10.1103/PhysRevD.93.055045. https:// doi.org/10.1103/PhysRevD.95.119902. arXiv:1511.07447 [hepph] (Erratum: Phys. Rev. D 95(11), 119902(2017))

58. C.-H. Chen, T. Nomura, H. Okada, Explanation of $B \rightarrow$ $K^{(*)} \ell^{+} \ell^{-}$and muon $g-2$, and implications at the LHC. Phys. Rev. D 94(11), 115005 (2016). https://doi.org/10.1103/ PhysRevD.94.115005. arXiv:1607.04857 [hep-ph]

59. S. Raby, A. Trautner, Vectorlike chiral fourth family to explain muon anomalies. Phys. Rev. D 97(9), 095006 (2018). https://doi. org/10.1103/PhysRevD.97.095006. arXiv:1712.09360 [hep-ph]

60. C.-W. Chiang, H. Okada, E. Senaha, Dark matter, muon $g-2$, electric dipole moments, and $Z \rightarrow \ell_{i}^{+} \ell_{j}^{-}$in a one-loop induced neutrino model. Phys. Rev. D 96(1), 015002 (2017). https://doi. org/10.1103/PhysRevD.96.015002. arXiv:1703.09153 [hep-ph]
61. C.-H. Chen, T. Nomura, H. Okada, Excesses of muon $g-$ $2, R_{D^{(*)}}$, and $R_{K}$ in a leptoquark model. Phys. Lett. B 774, 456-464 (2017). https://doi.org/10.1016/j.physletb.2017.10.005. arXiv:1703.03251 [hep-ph]

62. H. Davoudiasl, W.J. Marciano, Tale of two anomalies. Phys. Rev. D 98(7), 075011 (2018). https://doi.org/10.1103/PhysRevD.98. 075011. arXiv:1806.10252 [hep-ph]

63. J. Liu, C.E.M. Wagner, X.-P. Wang, A light complex scalar for the electron and muon anomalous magnetic moments. JHEP 03, 008 (2019). https://doi.org/10.1007/JHEP03(2019)008. arXiv:1810.11028 [hep-ph]

64. A.E. CárcamoHernández, S. Kovalenko, R. Pasechnik, I. Schmidt, Phenomenology of an extended IDM with loop-generated fermion mass hierarchies. Eur. Phys. J. C 79(7), 610 (2019). https://doi. org/10.1140/epjc/s10052-019-7101-0. arXiv:1901.09552 [hep$\mathrm{ph}]$

65. T. Nomura, H. Okada, Muon anomalous magnetic moment, $Z$ boson decays, and collider physics in multicharged particles. Phys. Rev. D 101(1), 015021 (2020). https://doi.org/10.1103/ PhysRevD.101.015021. arXiv:1903.05958 [hep-ph]

66. J. Kawamura, S. Raby, A. Trautner, Complete vectorlike fourth family and new $U(1)^{\prime}$ for muon anomalies. Phys. Rev. D 100(5), 055030 (2019). https://doi.org/10.1103/PhysRevD.100.055030. arXiv:1906.11297 [hep-ph]

67. M. Bauer, M. Neubert, S. Renner, M. Schnubel, A. Thamm, Axionlike particles, lepton-flavor violation, and a new explanation of $a_{\mu}$ and $a_{e}$. Phys. Rev. Lett. 124(21), 211803 (2020). https://doi. org/10.1103/PhysRevLett.124.211803. arXiv:1908.00008 [hep$\mathrm{ph}]$

68. X.-F. Han, T. Li, L. Wang, Y. Zhang, Simple interpretations of lepton anomalies in the lepton-specific inert two-Higgs-doublet model. Phys. Rev. D 99(9), 095034 (2019). https://doi.org/10. 1103/PhysRevD.99.095034. arXiv:1812.02449 [hep-ph]

69. B. Dutta, Y. Mimura, Electron $g-2$ with flavor violation in MSSM. Phys. Lett. B 790, 563-567 (2019). https://doi.org/10. 1016/j.physletb.2018.12.070. arXiv:1811.10209 [hep-ph]

70. M. Badziak, K. Sakurai, Explanation of electron and muon $g-2$ anomalies in the MSSM. JHEP 10, 024 (2019). https://doi.org/ 10.1007/JHEP10(2019)024. arXiv:1908.03607 [hep-ph]

71. G. Hiller, C. Hormigos-Feliu, D.F. Litim, T. Steudtner, Anomalous magnetic moments from asymptotic safety. Phys. Rev. D 102(7), 071901 (2020). https://doi.org/10.1103/PhysRevD.102. 071901. arXiv:1910.14062 [hep-ph]

72. A.E. CárcamoHernández, S.F. King, H. Lee, S.J. Rowley, Is it possible to explain the muon and electron $g-2$ in a $Z^{\prime}$ model? Phys. Rev. D 101(11), 115016 (2020). https://doi.org/10.1103/ PhysRevD.101.115016. arXiv:1910.10734 [hep-ph]

73. T. Appelquist, M. Piai, R. Shrock, Lepton dipole moments in extended technicolor models. Phys. Lett. B 593, 175180 (2004). https://doi.org/10.1016/j.physletb.2004.04.062. arXiv:hep-ph/0401114

74. J.M. Gerard, Fermion mass spectrum in SU(2)-L x U(1). Z. Phys. C 18, 145 (1983). https://doi.org/10.1007/BF01572477

75. J. Kubo, A. Mondragon, M. Mondragon, E. Rodriguez-Jauregui, The Flavor symmetry. Prog. Theor. Phys. 109, 795-807 (2003). https://doi.org/10.1143/PTP.109.795. arXiv:hep-ph/0302196 (Erratum: Prog. Theor. Phys.114, 287 (2005))

76. J. Kubo, Majorana phase in minimal S(3) invariant extension of the standard model. Phys. Lett. B 578, 156-164 (2004). https:// doi.org/10.1016/j.physletb.2005.06.013. https://doi.org/10.1016/ j.physletb.2003.10.048. arXiv:hep-ph/0309167 (Erratum: Phys. Lett. B 619, 387 (2005))

77. T. Kobayashi, J. Kubo, H. Terao, Exact S(3) symmetry solving the supersymmetric flavor problem. Phys. Lett. B 568, 83-91 (2003). https://doi.org/10.1016/j.physletb.2003.03.002. arXiv:hep-ph/0303084 
78. S.-L. Chen, M. Frigerio, E. Ma, Large neutrino mixing and normal mass hierarchy: a discrete understanding. Phys. Rev. D 70, 073008 (2004). https://doi.org/10.1103/PhysRevD.70.079905. https:// doi.org/10.1103/PhysRevD.70.073008. arXiv:hep-ph/0404084. (Erratum: Phys. Rev. D 70, 079905 (2004))

79. A. Mondragon, M. Mondragon, E. Peinado, Lepton masses, mixings and FCNC in a minimal S(3)-invariant extension of the Standard Model. Phys. Rev. D 76, 076003 (2007). https://doi.org/10. 1103/PhysRevD.76.076003. arXiv:0706.0354 [hep-ph]

80. A. Mondragon, M. Mondragon, E. Peinado, Lepton flavour violating processes in an S(3)-symmetric model. Rev. Mex. Fis. 54(3), 81-91 (2008). arXiv:0805.3507 [hep-ph] (Rev. Mex. Fis. Suppl. 54, $0181(2008)$ )

81. G. Bhattacharyya, P. Leser, H. Pas, Exotic Higgs boson decay modes as a harbinger of $S_{3}$ flavor symmetry. Phys. Rev. D 83, 011701 (2011). https://doi.org/10.1103/PhysRevD.83.011701. arXiv: 1006.5597 [hep-ph]

82. A.G. Dias, A.C.B. Machado, C.C. Nishi, An $S_{3}$ model for lepton mass matrices with nearly minimal texture. Phys. Rev. D 86, 093005 (2012). https://doi.org/10.1103/PhysRevD.86. 093005. arXiv:1206.6362 [hep-ph]

83. D. Meloni, $S_{3}$ as a flavour symmetry for quarks and leptons after the Daya Bay result on $\theta_{13}$. JHEP 05, 124 (2012). https://doi.org/ 10.1007/JHEP05(2012)124. arXiv:1203.3126 [hep-ph]

84. F. Gonzalez Canales, A. Mondragon, M. Mondragon, The $S_{3}$ flavour symmetry: neutrino masses and mixings. Fortsch. Phys. 61, 546-570 (2013). https://doi.org/10.1002/prop.201200121. arXiv: 1205.4755 [hep-ph]

85. F. González Canales, A. Mondragón, M. Mondragón, U.J. Saldaña Salazar, L. Velasco-Sevilla, Quark sector of S3 models: classification and comparison with experimental data. Phys. Rev. D 88, 096004 (2013). https://doi.org/10.1103/PhysRevD.88. 096004. arXiv:1304.6644 [hep-ph]

86. E. Ma, B. Melic, Updated $S_{3}$ model of quarks. Phys. Lett. B 725, 402-406 (2013). https://doi.org/10.1016/j.physletb.2013.07.015. arXiv:1303.6928 [hep-ph]

87. Y. Kajiyama, H. Okada, K. Yagyu, Electron/muon specific two Higgs doublet model. Nucl. Phys. B 887, 358370 (2014). https://doi.org/10.1016/j.nuclphysb.2014.08.009. arXiv:1309.6234 [hep-ph]

88. E. Ma, R. Srivastava, Dirac or inverse seesaw neutrino masses with $B-L$ gauge symmetry and $S_{3}$ flavor symmetry. Phys. Lett. B 741, 217-222 (2015). https://doi.org/10.1016/j.physletb.2014. 12.049. arXiv: 1411.5042 [hep-ph]

89. S. Gupta, C.S. Kim, P. Sharma, Radiative and seesaw threshold corrections to the $S_{3}$ symmetric neutrino mass matrix. Phys. Lett. B 740, 353-358 (2015). https://doi.org/10.1016/j.physletb.2014. 12.005. arXiv:1408.0172 [hep-ph]

90. A.E. Cárcamo Hernández, I. de Medeiros Varzielas, E. Schumacher, Fermion and scalar phenomenology of a two-Higgsdoublet model with $S_{3}$. Phys. Rev. D 93(1), 016003 (2016). https:// doi.org/10.1103/PhysRevD.93.016003. arXiv:1509.02083 [hep$\mathrm{ph}]$

91. A.E. Cárcamo Hernández, I. de Medeiros Varzielas, N.A. Neill, Novel Randall-Sundrum model with $S_{3}$ flavor symmetry. Phys. Rev. D 94(3), 033011 (2016). https://doi.org/10.1103/PhysRevD. 94.033011. arXiv:1511.07420 [hep-ph]

92. A.E. Cárcamo Hernández, A novel and economical explanation for SM fermion masses and mixings. Eur. Phys. J. C 76(9), 503 (2016). https://doi.org/10.1140/epjc/s10052-016-4351-y. arXiv:1512.09092 [hep-ph]

93. A.E. Cárcamo Hernández, I. de Medeiros Varzielas, E. Schumacher, The $750 \mathrm{GeV}$ diphoton resonance in the light of a $2 \mathrm{HDM}$ with $S_{3}$ flavour symmetry. arXiv:1601.00661 [hep-ph]
94. A.E. CárcamoHernández, S. Kovalenko, I. Schmidt, Radiatively generated hierarchy of lepton and quark masses. JHEP (2017). https://doi.org/10.1007/JHEP02(2017)125

95. C. Arbeláez, A.E. Cárcamo Hernández, S. Kovalenko, I. Schmidt, Radiative seesaw-type mechanism of fermion masses and nontrivial quark mixing. Eur. Phys. J. C 77(6), 422 (2017). https://doi. org/10.1140/epjc/s10052-017-4948-9. arXiv:1602.03607 [hep$\mathrm{ph}]$

96. J.C. Gómez-Izquierdo, Non-minimal flavored $S_{3} \otimes Z_{2}$ left-right symmetric model. Eur. Phys. J. C 77(8), 551 (2017). https://doi. org/10.1140/epjc/s10052-017-5094-0. arXiv:1701.01747 [hep$\mathrm{ph}]$

97. A.A. Cruz, M. Mondragón, Neutrino masses, mixing, and leptogenesis in an S3 model. arXiv:1701.07929 [hep-ph]

98. E. Ma, Cobimaximal neutrino mixing from $S_{3} \times Z_{2}$. Phys. Lett. B 777, 332-334 (2018). https://doi.org/10.1016/j.physletb.2017. 12.049. arXiv: 1707.03352 [hep-ph]

99. C. Espinoza, E.A. Garcés, M. Mondragón, H. Reyes-González, The $S 3$ symmetric model with a Dark scalar. Phys. Lett. B $\mathbf{7 8 8}$, 185-191 (2019). https://doi.org/10.1016/j.physletb.2018.11.028. arXiv: 1804.01879 [hep-ph]

100. E.A. Garcés, J.C. Gómez-Izquierdo, F. Gonzalez-Canales, Flavored non-minimal left-right symmetric model fermion masses and mixings. Eur. Phys. J. C 78(10), 812 (2018). https://doi.org/ 10.1140/epjc/s10052-018-6271-5. arXiv:1807.02727 [hep-ph]

101. A.E. CárcamoHernández, J. Vignatti, A. Zerwekh, Generating lepton masses and mixings with a heavy vector doublet. J. Phys. G 46(11), 115007 (2019). https://doi.org/10.1088/1361-6471/ ab4499. arXiv:1807.05321 [hep-ph]

102. J.C. Gómez-Izquierdo, M. Mondragón, B-L model with $\mathbf{S}_{3}$ symmetry: nearest neighbor interaction textures and broken $\mu \leftrightarrow \tau$ symmetry. Eur. Phys. J. C 79(3), 285 (2019). https://doi.org/10. 1140/epjc/s10052-019-6785-5. arXiv:1804.08746 [hep-ph]

103. S. Pramanick, Scotogenic S3 symmetric generation of realistic neutrino mixing. Phys. Rev. D 100(3), 035009 (2019). https://doi. org/10.1103/PhysRevD.100.035009. arXiv:1904.07558 [hep-ph]

104. C. Salazar, R.H. Benavides, W.A. Ponce, E. Rojas, LHC constraints on 3-3-1 models. JHEP 07, 096 (2015). https://doi.org/ 10.1007/JHEP07(2015)096. arXiv:1503.03519 [hep-ph]

105. V.T.N. Huyen, H.N. Long, T.T. Lam, V.Q. Phong, Neutral current in reduced minimal 3-3-1 model. Commun. Phys. 24(2), 97 (2014). https://doi.org/10.15625/0868-3166/24/ 2/377410.15625/0868-3166/24/2/3774. arXiv:1210.5833 [hep$\mathrm{ph}]$

106. R. Martinez, F. Ochoa, Mass-matrix ansatz and constraints on B0(s)-anti-B0(s) mixing in 331 models. Phys. Rev. D 77, 065012 (2008). https://doi.org/10.1103/PhysRevD.77.065012. arXiv:0802.0309 [hep-ph]

107. A.J. Buras, F. De Fazio, J. Girrbach, 331 models facing new $b \rightarrow s \mu^{+} \mu^{-}$data. JHEP 02, 112 (2014). https://doi.org/10.1007/ JHEP02(2014)112. arXiv:1311.6729 [hep-ph]

108. A.J. Buras, F. De Fazio, J. Girrbach-Noe, $Z-Z^{\prime}$ mixing and $Z$-mediated FCNCs in $S U(3)_{C} \times S U(3)_{L} \times U(1)_{X}$ models. JHEP 08, 039 (2014). https://doi.org/10.1007/JHEP08(2014)039. arXiv:1405.3850 [hep-ph]

109. A.J. Buras, F. De Fazio, J. Girrbach, M.V. Carlucci, The anatomy of quark flavour observables in 331 models in the flavour precision era. JHEP 02, 023 (2013). https://doi.org/10.1007/ JHEP02(2013)023. arXiv:1211.1237 [hep-ph]

110. J. Kubo, H. Okada, F. Sakamaki, Higgs potential in minimal S(3) invariant extension of the standard model. Phys. Rev. D 70, 036007 (2004). https://doi.org/10.1103/PhysRevD.70. 036007. arXiv:hep-ph/0402089

111. K. Hagiwara, R. Liao, A.D. Martin, D. Nomura, T. Teubner, $(g-2)_{\mu}$ and $\alpha\left(M_{Z}^{2}\right)$ re-evaluated using new precise data. J. Phys. 
G 38, 085003 (2011). https://doi.org/10.1088/0954-3899/38/8/ 085003. arXiv:1105.3149 [hep-ph]

112. M. Davier, A. Hoecker, B. Malaescu, Z. Zhang, Reevaluation of the hadronic vacuum polarisation contributions to the Standard Model predictions of the muon $g-2$ and $\alpha\left(m_{Z}^{2}\right)$ using newest hadronic cross-section data. Eur. Phys. J. C 77(12), 827 (2017). https://doi.org/10.1140/epjc/s10052-017-5161-6. arXiv:1706.09436 [hep-ph]

113. T. Nomura, H. Okada, One-loop neutrino mass model without any additional symmetries. Phys. Dark Univ. 26, 100359 (2019). https://doi.org/10.1016/j.dark.2019.100359. arXiv:1808.05476 [hep-ph]

114. T. Nomura, H. Okada, Zee-Babu type model with $U(1)_{L_{\mu}-L_{\tau}}$ gauge symmetry. Phys. Rev. D 97(9), 095023 (2018). https://doi. org/10.1103/PhysRevD.97.095023. arXiv:1803.04795 [hep-ph]

115. RBC, UKQCD Collaboration, T. Blum, P. A. Boyle, V. Gülpers, T. Izubuchi, L. Jin, C. Jung, A. Jüttner, C. Lehner, A. Portelli, J.T. Tsang, Calculation of the hadronic vacuum polarization contribution to the muon anomalous magnetic moment. Phys. Rev. Lett. 121(2), 022003 (2018). https://doi.org/10.1103/PhysRevLett. 121.022003. arXiv:1801.07224 [hep-lat]

116. A. Keshavarzi, D. Nomura, T. Teubner, Muon $g-2$ and $\alpha\left(M_{Z}^{2}\right)$ : a new data-based analysis. Phys. Rev. D 97(11), 114025 (2018). https://doi.org/10.1103/PhysRevD.97.114025. arXiv:1802.02995 [hep-ph]

117. T. Aoyama et al., The anomalous magnetic moment of the muon in the Standard Model. Phys. Rep. 887, 1-166 (2020). https://doi. org/10.1016/j.physrep.2020.07.006. arXiv:2006.04822 [hep-ph]

118. R.H. Parker, C. Yu, W. Zhong, B. Estey, H. Müller, Measurement of the fine-structure constant as a test of the Standard Model. Science 360, 191 (2018). https://doi.org/10.1126/science.aap7706. arXiv:1812.04130 [physics.atom-ph]

119. W. Grimus, L. Lavoura, The seesaw mechanism at arbitrary order: disentangling the small scale from the large scale. JHEP 11, 042 (2000). https://doi.org/10.1088/1126-6708/2000/11/042. arXiv:hep-ph/0008179

120. R.A. Diaz, R. Martinez, J.A. Rodriguez, Phenomenology of lepton flavor violation in $2 \mathrm{HDM}(3)$ from $(\mathrm{g}-2)(\mathrm{mu})$ and leptonic decays. Phys. Rev. D 67, 075011 (2003). https://doi.org/10.1103/ PhysRevD.67.075011. arXiv:hep-ph/0208117

121. M. Lindner, M. Platscher, F.S. Queiroz, A call for new physics: the muon anomalous magnetic moment and lepton flavor violation. Phys. Rep. 731, 1-82 (2018). https://doi.org/10.1016/j.physrep. 2017.12.001. arXiv:1610.06587 [hep-ph]

122. K. Kowalska, E.M. Sessolo, Expectations for the muon g-2 in simplified models with dark matter. JHEP 09, 112 (2017). https:// doi.org/10.1007/JHEP09(2017)112. arXiv:1707.00753 [hep-ph]

123. K. Bora, Updated values of running quark and lepton masses at GUT scale in SM, 2HDM and MSSM. Horizon 2, 112 (2013). arXiv:1206.5909 [hep-ph]

124. Z.-Z. Xing, H. Zhang, S. Zhou, Updated values of running quark and lepton masses. Phys. Rev. D 77, 113016 (2008). https://doi. org/10.1103/PhysRevD.77.113016. arXiv:0712.1419 [hep-ph]

125. Particle Data Group Collaboration, M. Tanabashi et al., Review of Particle Physics. Phys. Rev. D 98(3), 030001 (2018). https:// doi.org/10.1103/PhysRevD.98.030001

126. E. Ma, Verifiable radiative seesaw mechanism of neutrino mass and dark matter. Phys. Rev. D 73, 077301 (2006). https://doi.org/ 10.1103/PhysRevD.73.077301. arXiv:hep-ph/0601225

127. H. Ishimori, T. Kobayashi, H. Ohki, Y. Shimizu, H. Okada, M. Tanimoto, Non-Abelian discrete symmetries in particle physics. Prog. Theor. Phys. Suppl. 183, 1-163 (2010). https://doi.org/10. 1143/PTPS.183.1. arXiv:1003.3552 [hep-th] 